1 Combination of sirtuin 3 and hyperoxia diminishes tumorigenic properties of MDA-MB-

3 Iva I. Podgorski ${ }^{\mathrm{a}, \dagger}, *$, Marija Pinteric ${ }^{a}{ }^{\dagger}$, , Dora Marčinko ${ }^{\mathrm{a}}$, Marijana Popović Hadžija ${ }^{\mathrm{a}}$, Vedrana

4 Filić $^{b}$, Ivan Ciganek ${ }^{a}$, Denis Pleše ${ }^{a}$, Tihomir Balog $^{a}$, Sandra Sobočanec $^{\mathrm{a},{ }^{+}}$

5 aDivision of Molecular Medicine, Ruđer Bošković Institute, Zagreb, Croatia

6 bivision of Molecular Biology, Ruđer Bošković Institute, Zagreb, Croatia

$7 \quad$ *Corresponding author: ivapodgorski@gmail.com, +385 1456 1064, Ruđer Bošković

8 Institute, Bijenička cesta 54, 10000 Zagreb

$9+$ equal contribution

\title{
11 Acknowledgements
}

12 This work was supported by the Croatian Science Foundation (HRZZ) [grant number IP-201413 09-4533]. The authors would like to thank Iva Pešun Međimorec and Marina Marš for their 14 excellent technical contribution.

15 Conflict of Interest statement: The authors declare that there are no conflicts of interest. 


\section{Abstract}

18 Aims: Since the role of the major mitochondrial NAD+-dependent deacetylase, sirtuin 3 (Sirt3), 19 is differential in cancer, opposite to the well-known tumor-suppressing effect of hyperoxia, 20 this study aimed to investigate the role of Sirt3 in triple-negative breast cancer (TNBC) cell 21 line MDA-MB-231 upon hyperoxic $\left(95 \% \mathrm{O}_{2}\right)$ conditions.

22 Main methods: MDA-MB-231 cells were stably transfected with Flag-tagged Sirt-3 or empty 23 plasmid. Western blot and real-time PCR were used to monitor the expression of proteins or 24 genes involved in mitochondrial biogenesis, metabolic regulation and antioxidant defense. 25 Immunocytochemistry and confocal microscopy were used to confirm the cellular localization 26 and abundance of proteins. Flow cytometry was used to analyze mitochondrial mass, 27 potential and ROS production, and MTT test as a measure of metabolic activity. Mitotic index 28 analysis, colony-forming unit assay, DNA damage and Annexin V-FITC analyses were used to 29 assess the differences in the growth and apoptosis rate.

30 Key findings: Although Sirt3 seemed to improve mitochondrial properties by increasing 31 mitochondrial mass and potential, metabolic activity (Warburg effect) and antioxidative 32 defense (SOD2, Cat), it also increased mitochondrial ROS, induced DNA damage, timp-1 33 expression, formation of multinucleated cells and apoptosis, and finally markedly reduced the 34 proliferation of MDA-MB-231 cells. All these effects were even more evident upon the 35 hyperoxic treatment, thus pointing towards combined negative effect of Sirt3 and hyperoxia 36 on MDA-MB-231 cells.

37 Significance: Both Sirt3 and hyperoxia, alone or in combination, have the potential to 38 negatively affect the malignant properties of the MDA-MB-231 cells and should be further explored as a possible therapy for TNBC.

Keywords: sirtuin 3, MDA-MB-231, breast cancer, oxidative stress, hyperoxia

42 Word count: 5965

43 Figure/table count: 8 figures, 2 tables

\section{Introduction}

45 Breast cancer is the most common malignant tumor in women and the third cause of death 46 in the world [1]. Numerous studies focus on triple-negative breast cancer (TNBC) cells, which 47 are more aggressive and resistant to endocrine therapy since they lack the expression of 48 estrogen and progesterone receptor and human epidermal growth factor type 2 [2]. 10-15\% 49 of breast cancer patients suffer from TNBC and have a much worse treatment outcome 50 compared to other breast cancer subtypes [3]. For this reason, there has been an interest in 51 further research at the molecular level to obtain a more accurate diagnosis and possible 52 treatment therapies. 
53 Oxidative stress occurs as a result of high levels of reactive oxygen species (ROS) and is defined

54 as a disturbance of the balance between ROS production and antioxidant defense $[4,5]$. 55 Numerous studies confirm that tumor cells typically exhibit higher levels of ROS than healthy 56 cells, and high levels of ROS induce DNA damage and genomic instability, resulting in the 57 continued growth and proliferation of tumor cells, as well as resistance to chemotherapy [658 8]. In addition to being a consequence of common biochemical processes, ROS are products 59 of increased oxygen concentration in the cell caused by hyperoxia. Thereby, hyperoxia60 induced ROS levels could be certainly toxic for cancer cells and could impair tumor growth, 61 i.e. reduce tumor aggressive behavior $[9,10]$. Also, some studies have confirmed a smaller 62 number of TNBC metastases under hyperoxic conditions [11].

63 The mammalian sirtuin (Sirt) family is described with seven proteins (Sirt1-7), three of which 64 are the mitochondrial sirtuins (Sirt3-5) [12]. Sirt3 is a major mitochondrial NAD+-dependent 65 deacetylase with an essential role in maintaining energy homeostasis [13]. In the absence of 66 Sirt3 the mitochondrial proteins become hyperacetylated, thus reducing the mitochondrial 67 ability to generate ATP and elevating oxidative stress (reviewed in [14]). As a defense strategy 68 against oxidative damage caused by ROS, the cells most commonly use the enzymatic 69 antioxidants superoxide dismutase (SOD), catalase (Cat), glutathione peroxidase and 70 glutathione reductase [15]. Sirt3 plays a key role in the deacetylation and activation of some 71 of these antioxidant enzymes, with SOD2 as one of the main targets. However, the role of 72 Sirt3 in breast cancer is still controversial [8].

73 Due to the known tumor-suppressing effect of hyperoxic treatment and a differential role of 74 Sirt3 in cancer, this study aimed to investigate the role of increased expression of the Sirt3 75 upon hyperoxia on morphology, proliferation, cell cycle, metabolic activity, DNA damage, 76 apoptosis and mitochondrial function of TNBC cells MDA-MB-231. We found that, although

77 Sirt3 seemed to improve several mitochondrial properties and antioxidative defense, it also 78 increased mitochondrial ROS, induced DNA damage, apoptosis, formation of multinucleated 79 cells, and significantly diminished the proliferation of MDA-MB-231 cells. These effects were 80 even more pronounced upon the hyperoxic treatment, thus pointing towards a combined 81 negative effect of Sirt3 and hyperoxia on MDA-MB-231 cells. Therefore, both Sirt3 and 82 hyperoxia have the potential to negatively modulate the malignant properties of the MDA83 MB-231 cells and should be further explored as a possible therapy for TNBC.

\section{Materials and Methods}

\section{Cell culture and transfection}

86 In the present study, adherent, epithelial, basal-like triple-negative breast cancer cell line 87 MDA-MB-231 (ATCC ${ }^{\circledR}$ HTB-26 ${ }^{\text {TM }}$; Manassas, VA, USA) was used. Cells were transfected with 88 the pcDNA3.1+ Flag-Sirt3 plasmid containing the Flag-tagged Sirt3 gene (Addgene 89 Cat.No.13814) as described previously [16], and are marked as MDA-S3. Cells transfected with 90 the empty plasmid pcDNA3.1+ were used as control (MDA-C). The cells were grown in 
91 complete high glucose (4.5 g/L) Dulbecco's modified Eagle's medium (DMEM; Sigma Aldrich,

92 St. Louis, MO, USA) with the addition of $10 \%$ fetal bovine serum (FBS; Capricorn Scientific $93 \mathrm{GmbH}$, Ebsdorfergrund, Germany), 1\% non-essential amino acids (Capricorn Scientific $\mathrm{GmbH}$,

94 Ebsdorfergrund, Germany) and 1\% antibiotic/antimycotic solution (Capricorn Scientific $95 \mathrm{GmbH}$, Ebsdorfergrund, Germany).

Normoxic and hyperoxic conditions

97 After seeding, the cells were allowed to adhere for $24 \mathrm{~h}$ and then treated in normoxic or 98 hyperoxic conditions $\left(95 \% \mathrm{O}_{2}\right)$. To achieve hyperoxic conditions, cells were kept in a self99 contained and sealed hyperoxic chamber (StemCellTM Technology Inc., Vancouver, Canada) 100 for $16 \mathrm{~h}$ in an incubator at $37^{\circ} \mathrm{C}$, together with control cells (normoxia) under standard 101 conditions $\left(37^{\circ} \mathrm{C}, 5 \% \mathrm{CO} 2\right)$.

\section{RNA isolation and real-time PCR}

103 After treatment (hyperoxic and normoxic), the cells were collected in Trizol Reagent (Life 104 Technologies, Carlsbad, CA, USA) for RNA extraction according to the manufacturer's 105 recommendations. The RNA was quantified using the Nanodrop (NanoPhotometer N60/N50, 106 Implen $\mathrm{GmbH}$, München, Germany) and treated with DNAase (TURBO DNA-free Kit, Thermo 107 Fisher Scientific, Waltham, MA, USA), followed by reverse transcription (Applied Biosystems ${ }^{\mathrm{TM}}$ 108 High-Capacity cDNA Reverse Transcription Kit; Foster City, CA, USA). Taqman assays (Thermo 109 Fisher Scientific, Waltham, MA, USA) for sirt3 (Hs00953477_m1), timp-1 (Hs01092512_g1) 110 and B-actin (Hs01060665_g1) genes were used for real-time PCR performed on a 7300 Real111 time PCR system device (Applied Biosystems, Foster City, CA, USA) using a comparative CT (2$112 \Delta \Delta C t)$ method to determine the relative gene expression level.

\section{Protein isolation and Western blot}

114 After hyperoxic treatment, cells were trypsinized (Capricorn Scientific, Ebsdorfergrund, 115 Germany), collected and centrifuged, and the cell pellet was resuspended in RIPA buffer (50 $116 \mathrm{mM}$ Tris buffer, $150 \mathrm{mM} \mathrm{NaCl}, 0.1 \%$ SDS, $12 \mathrm{mM}$ Na-deoxycholate, 1\% Triton) with protease 117 inhibitors (cOmpleteTM EDTA-free EASYpack, Roche, Basel, Switzerland). The lysate was 118 sonicated (amplitude 80\%; Labsonic, Biotech International Ltd., Delhi, India) and centrifuged 119 for $20 \mathrm{~min}$ at $16000 \mathrm{~g}\left(4^{\circ} \mathrm{C}\right)$ and the supernatant was transferred to a new tube, from which 120 the protein concentration was measured with Pierce ${ }^{\mathrm{TM}}$ BCA Protein Assay Kit (Thermo Fischer 121 Scientific, Waltham, MA, USA). The proteins were prepared in SDS-PAGE sample buffer (100 $122 \mathrm{mM}$ Tris- $\mathrm{HCl}(\mathrm{pH} \mathrm{6,8),2 \%} \mathrm{SDS,} \mathrm{20 \%} \mathrm{glycerol,} 4 \% \beta$-mercaptoethanol, 0,5\% bromophenol blue 123 dye) and Western blot analysis was carried out as described previously [16]. Antibodies used 124 in this study are listed in Table 1.

125 Table 1. Antibodies used for Western blot analyses

\begin{tabular}{|l|l|l|l|}
\hline Antibody & Dilution & Host & Manufacturer \\
\hline
\end{tabular}




\begin{tabular}{|l|l|l|l|}
\hline Sirt3 (F-10, sc-365175) & $1: 500$ & Mouse & $\begin{array}{l}\text { Santa Cruz } \\
\text { Biotechnology, USA }\end{array}$ \\
\hline Catalase (ab1877) & $1: 1000$ & Rabbit & Abcam, UK \\
\hline $\begin{array}{l}\text { Gamma H2A.X (phospho S139, } \\
\text { ab11174) }\end{array}$ & $1: 8000$ & Rabbit & Abcam, UK \\
\hline SOD2 (ab13533) & $1: 2000$ & Rabbit & Abcam, UK \\
\hline Sirt1 (NBP2-27205) & $1: 300$ & Rabbit & Novus Biologicals, USA \\
\hline Ldh-A (sc-33781) & $1: 200$ & Rabbit & $\begin{array}{l}\text { Santa Cruz } \\
\text { Biotechnology, USA }\end{array}$ \\
\hline PGC-1 $\alpha$ (NBP1-04676) & $1: 1000$ & Rabbit & Novus Biologicals, USA \\
\hline Anti-mouse (170-6516) & $1: 5000$ & Goat & Bio-rad, USA \\
\hline Anti-rabbit (NA934) & $1: 5000$ & Goat & GE Healthcare, USA \\
\hline
\end{tabular}

\section{MTT and CFU assays}

128 For the MTT assay, 5000 cells were seeded into 96-well plates. After hyperoxic treatment, the 129 plates were left for an additional $48 \mathrm{~h}$ in the incubator under normal conditions and then 130 processed as described previously [16]. For the colony-forming unit (CFU) assay, 1500 cells 131 were seeded per Petri dish $(6 \mathrm{~cm})$. After hyperoxic treatment, the cells were left for 10 days 132 until visible colonies were formed and the plates were processed as described previously [16].

\section{Flow cytometry analysis of mitochondrial parameters, ROS and apoptosis}

134 Mitotracker Deep Red dye (100 nM; Thermo Fisher Scientific, Waltham, MA, USA) was used 135 for mitochondrial membrane potential $(\Delta \Psi)$ analysis, and $N A O(0,5 \mathrm{nM} ; 10-\mathrm{N}$-nonyl acridine 136 orange, Invitrogen, Carlsbad, CA, USA) for mitochondrial mass. DHE dye (20 $\mu \mathrm{M}$; 137 dihydroethidium, Sigma Aldrich, St. Louis, MO, USA) was used to measure cytosolic ROS and 138 MitosoxRed (5 $\mu \mathrm{M}$; Thermo Fisher Scientific, Waltham, MA, USA) to detect mitochondrial 139 ROS. ApoScreen ${ }^{\mathrm{TM}}$ Annexin V Apoptosis Kit (SouthernBiotech, Birmingham, AL, USA) was used 140 according to the manufacturer's protocol for apoptosis analysis. After hyperoxic treatment, 141 the cells were trypsinized and collected, washed with $1 \mathrm{x}$ PBS, distributed (300000 142 cells/sample) into FACS tubes, resuspended in each dye and incubated for $30 \mathrm{~min}$ at $37^{\circ} \mathrm{C}$ 143 (those for apoptosis $15 \mathrm{~min}$ in dark at room temperature (RT) followed by analysis on FACS 144 within $1 \mathrm{~h})$. After the incubation, all the cells except those in the DHE solution were 145 centrifuged, washed with 1x PBS and resuspended in 1x PBS + 1\% FCS. Propidium iodide (PI) 146 at a concentration of $1.5 \mu \mathrm{g} / \mathrm{mL}$ was added to tubes with NAO and Mitotracker Deep Red to 147 exclude dead cells, whereas 500 nM Sytox Red (Thermo Fisher Scientific, Waltham, MA, USA) 148 was used with DHE and MitosoxRed for the same purpose. The samples were analyzed on a 149 flow cytometer (FACSCalibur, BD Biosciences, Franklin Lakes, NJ, USA) in FL1 (NAO and 150 annexin), FL2 (DHE and MitosoxRed), FL3 (PI) and FL4 (Mitotracker Deep Red and Sytox Red) 151 channel. The data analysis was performed using the FCS Express 7 software package (De Novo 152 software, Pasadena, CA, USA). 
Mitotic index analysis

154 After hyperoxic treatment, the medium from the cells was collected in tubes, the cells were 155 trypsinized and collected by pooling with appropriate medium, washed twice with $1 \mathrm{x}$ PBS and 156 resuspended in cold $1 x$ PBS, followed by dropwise addition of $-20^{\circ} \mathrm{C}$ pre-cooled $96 \% \mathrm{EtOH}$ on 157 a mild vortex. Samples were stored at $-20^{\circ} \mathrm{C}$ until the day of the experiment (minimum for 6 158 h). Samples were centrifuged and the cell pellet was washed with 1x PBS, centrifuged again, 159 and the resulting pellets were resuspended in $1 \mathrm{x}$ buffer ( $1 \%$ FCS and $0.1 \%$ Triton X-100 in 1x 160 PBS), centrifuged, and resuspended in primary anti-phosho-Histone H3 (Ser 10) antibody 161 (1:200; Cat. No. 06-570; Merck Millipore, Burlington, MA, USA) prepared in 1x buffer and 162 incubated for $1 \mathrm{~h}$ at RT. Afterward the pellets were washed with $1 \mathrm{x}$ buffer and incubated for $1631 \mathrm{~h}$ at RT in dark with a secondary antibody (Alexa488, 1:1000; A-11001, Thermo Fisher 164 Scientific, Waltham, MA, USA), followed by two washes with $1 x$ buffer. Cells were finally 165 resuspended in $0.1 \mu \mathrm{g} / \mu \mathrm{L}$ RNase A (iNtRON Biotechnology, Seongnam, South Korea) and 50 $166 \mu \mathrm{g} / \mu \mathrm{L}$ propidium iodide (PI) solution, and FACS analysis was performed (FACSCalibur, BD 167 Biosciences, Franklin Lakes, NJ, USA).

\section{SOD activity analysis}

169 For superoxide dismutase (SOD) activity analysis, the RANSOD kit (RANDOX Labs, Crumlin, UK) 170 was used according to the manufacturer's protocol, as described previously [17].

\section{Immunocytochemistry, fluorescence and confocal microscopy analysis}

172 Immunocytochemistry staining was performed as described previously $[16,17]$. MitoTracker 173 Deep Red (100 nM; Thermo Fisher Scientific, Waltham, MA, USA) was added after hyperoxic 174 treatment and cells were incubated for $20 \mathrm{~min}$ at $37^{\circ} \mathrm{C}$. Primary and secondary antibodies 175 used for immunocytochemistry in this study are listed in Table 2. Confocal imaging was 176 performed using Leica TCS SP8 X laser scanning microscope (Leica Microsystems, Wetzlar, 177 Germany), equipped with an HC PL APO CS2 63/1.40 oil immersion objective and a white light 178 laser. The excitation wavelengths and emission detection ranges used were $405 \mathrm{~nm}$ and $412-$ $179460 \mathrm{~nm}$ for DAPI, $488 \mathrm{~nm}$ and $495-550 \mathrm{~nm}$ for Alexa488, $594 \mathrm{~nm}$ and 601-644 nm for Alexa594, 180 and $644 \mathrm{~nm}$ and 651-700 nm for MitoTracker Deep Red, respectively. For cell morphology 181 analysis on EVOS Floid Cell Imaging Station (Thermo Fisher Scientific, USA), live cells in the 182 medium were stained with NAO $(1,5 \mu \mathrm{M})$ and Hoechst $33342(5 \mu \mathrm{g} / \mathrm{ml}$; Thermo Fisher 183 Scientific, USA) for 5 min, washed $2 x$ with $1 x$ PBS and maintained in $1 x$ PBS+Mg/Ca.

Table 2. Antibodies used for immunofluorescence analyses

\begin{tabular}{|l|l|l|l|}
\hline Antibody & Dilution & Host & Manufacturer \\
\hline Sirt3 (F-10, sc-365175) & $1: 100$ & Mouse & Santa Cruz Biotechnology, USA \\
\hline $\begin{array}{l}\text { Gamma H2A.X (phospho } \\
\text { S139, ab11174) }\end{array}$ & $1: 5000$ & Rabbit & Abcam, UK \\
\hline
\end{tabular}




\begin{tabular}{|l|l|l|l|}
\hline $\begin{array}{l}\text { Anti-mouse Alexa } 488 \text { (A- } \\
\text { 11001) }\end{array}$ & $1: 2000$ & Goat & Thermo Fisher Scientific, USA \\
\hline $\begin{array}{l}\text { Anti-rabbit Alexa } 594 \text { (A- } \\
\text { 11012) }\end{array}$ & $1: 1000$ & Goat & Thermo Fisher Scientific, USA \\
\hline
\end{tabular}

\section{Statistical analysis}

187 Statistical analysis of data was performed using SPSS (17.0, IBM, Armonk, NY, USA) for

188 Windows as described previously [17]. Significance is set at $p<0.05$.

189 Results

190 Overexpressed Sirt-3 is localized in mitochondria and its expression is increased upon

191 hyperoxic treatment in MDA-MB-231 cells. MDA-MB-231 cells with stably transfected 192 pcDNA3.1+ Flag-Sirt3 plasmid were defined as MDA-S3, and cells with stably transfected 193 empty plasmid pcDNA3.1+ as MDA-C (control). Since Sirt3 is a major mitochondrial NAD+194 dependent deacetylase [13], first we aimed to confirm its expression and localization in the 195 transfected cells. Confocal microscopy analysis confirmed overexpressed Sirt3 and its 196 localization in mitochondria of MDA-S3 cells, unlike MDA-C cells (Figure 1A). The results of 197 the qPCR analysis showed that MDA-S3 cells have a 19-fold higher expression of the sirt3 gene 198 than MDA-C in normoxia, and as much as 38-fold higher in hyperoxia (Figure 1B). Western 199 blot analysis did not detect Sirt3 protein expression in the MDA-C cells, while it was visible in 200 the MDA-S3 as a $28 \mathrm{kDa}$ band (Figure 1C, D), with a stronger signal in hyperoxia, which is 201 consistent with the results of gene expression. Thus, we can conclude that Sirt3 was 202 successfully transfected into MDA-MB-231 cells, and that its expression is upregulated in 203 hyperoxic conditions. 


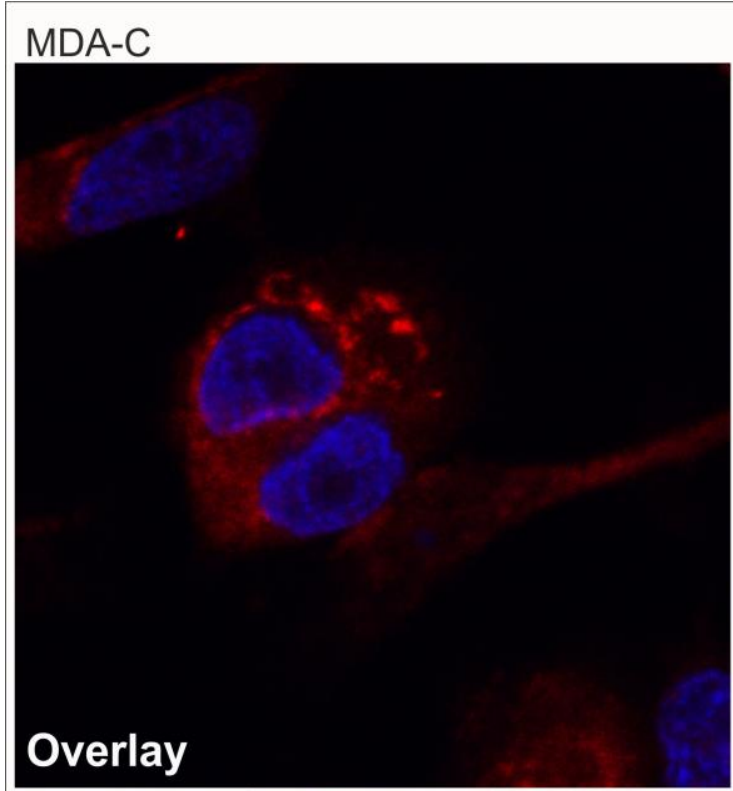

\section{MDA-S3}
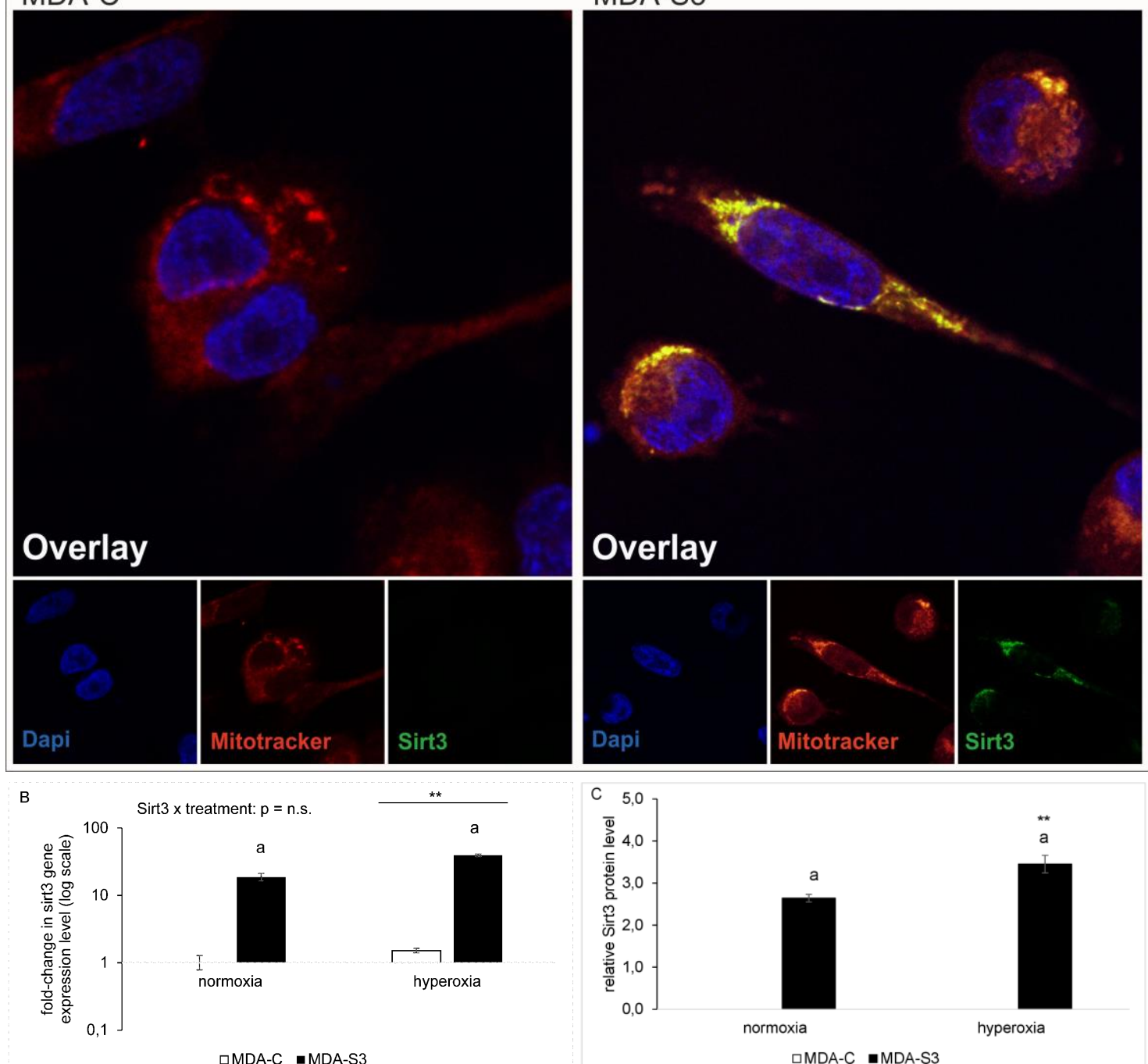

口MDA-C $=$ MDA-S3

口MDA-C $=$ MDA-S3

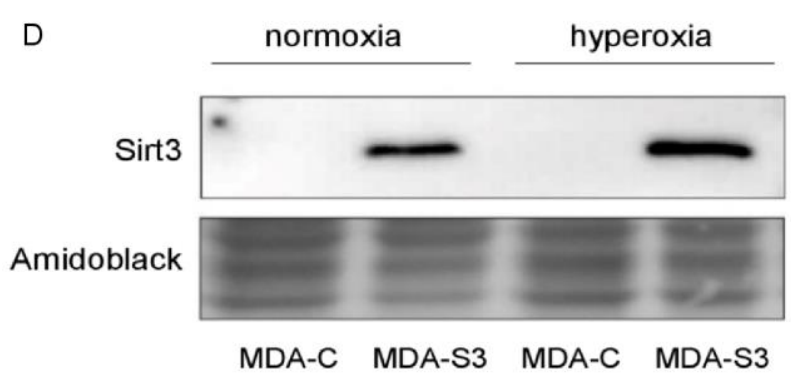

Figure 1. Overexpressed Sirt-3 is localized in mitochondria and its expression is increased upon hyperoxic treatment in MDA-MB-231 cells. (A) Representative confocal image of Sirt3 (green) in mitochondria (red; Mitotracker Deep Red). Nuclei were detected with DAPI (blue). Overlay image shows overlap (yellow) of the Sirt3 and mitochondria staining. (B) Real-time PCR analysis of sirt3 gene expression in MDA-C and MDA-S3 cells under normoxic and hyperoxic conditions: MDA-S3 vs. MDA-C $\left({ }^{a} p<0.001\right)$, hyperoxia vs. normoxia $(* * p<0.01)$. Data are presented as fold-change \pm S.E., and normalized to control group. $\beta$-actin was used as the endogenous control. 
(C) Densitometry analysis of Sirt3 protein expression in MDA-C and MDA-S3 cells under normoxic and hyperoxic conditions: MDA-S3 vs. MDA-C ( $\left.{ }^{a} p<0.001\right), M D A-S 3$ in hyperoxia vs. normoxia $\left({ }^{* *} p<0.01\right)$. Data are presented as mean \pm S.D. (D) Representative immunoblot of Sirt3 protein expression in MDA-C and MDA-S3 cells under normoxic and hyperoxic conditions. Amido black was used as a loading control. Experiments were repeated at least three times and representative data are shown.

Overexpressed Sirt-3 is associated with hyperoxia-induced alterations in mitochondrial mass and potential in MDA-MB-231 cells. Since Sirt3 is a mitochondrial protein involved in the regulation of mitochondrial mass and potential $[18,19]$, these parameters were analyzed by flow cytometry with the use of NAO and Mitotracker Deep Red dyes, respectively. Sirt3 expression caused an increase of mitochondrial mass in normoxia, which was further enhanced by hyperoxic treatment (Figure $\mathbf{2 A}$ ). On the other hand, higher mitochondrial potential in MDA-S3 cells in normoxia was reduced in both control and MDA-S3 cells upon hyperoxia (Figure 2B). Thus, we can conclude that Sirt3 increases both mitochondrial mass and potential, whereas hyperoxia has an inducing effect on mitochondrial mass and reducing effect on mitochondrial potential.

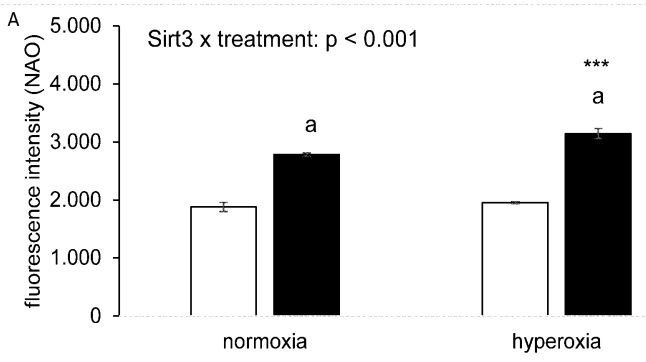

口MDA-C $=$ MDA-S3

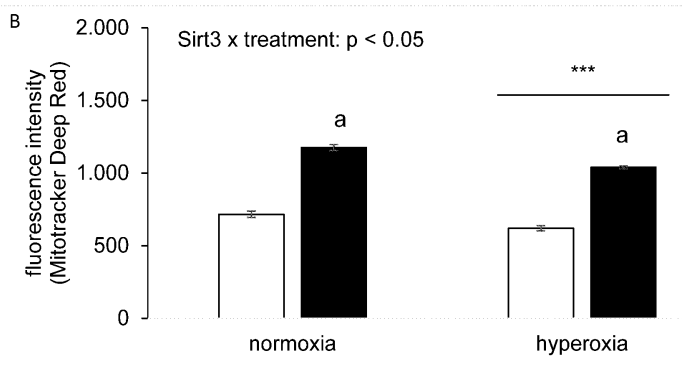

口MDA-C $\square$ MDA-S3

Figure 2. Overexpressed Sirt-3 is associated with hyperoxia-induced alterations in mitochondrial mass and potential in MDA-MB-231 cells. (A) Mitochondrial mass of MDA-C and MDA-S3 cells under normoxic and hyperoxic conditions measured with NAO dye: MDA-S3 vs. MDA-C $\left({ }^{\mathrm{a}} \mathrm{p}<0.001\right)$, MDA-S3 hyperoxia vs. normoxia $\left({ }^{* * *} p<0.001\right)$. (B) Mitochondrial potential of MDA-C and MDA-S3 cells under normoxic and hyperoxic conditions measured with Mitotracker Deep Red dye: MDA-S3 vs. MDAC $\left({ }^{a} p<0.001\right)$, hyperoxia vs. normoxia $\left({ }^{* * *} p<0.001\right)$. Data show the relative fluorescence intensity as the average geometric mean \pm SD. Experiments were repeated at least three times and representative data are shown.

Overexpressed Sirt3 affects metabolic activity and proteins involved in metabolism with the reverse effect of hyperoxic treatment on Ldh-A expression in MDA-MB-231 cells. Because in our study Sirt3 increased several mitochondrial parameters, we next analyzed the metabolic activity of transfected cells by MTT test and expression of several proteins involved in metabolic regulation (Sirt1, Ldh-A, PGC-1 $\alpha$ ). The MTT test results showed an increase of 
MDA-C cells (Figure $3 A$ ), which was accompanied by a similar pattern of Sirt1 expression (Figure 3B, E), a protein involved in the regulation of metabolic responses as well as in DNA damage response $[20,21]$. The observed higher metabolic activity of MDA-S3 cells was in normoxic conditions also followed by increased expression of lactate dehydrogenase (Ldh-A;

253 Figure 3C, E), a marker of aerobic glycolysis [22]. However, hyperoxic treatment had the opposite effect on its expression, thus MDA-S3 cells had lower Ldh-A expression than MDA-C cells. Expression of peroxisome proliferator-activated receptor gamma coactivator 1-alpha (PGC-1 $\alpha$ ), one of the key regulators of energy metabolism [23], was lower in MDA-S3 cells in both normoxia and hyperoxia compared to MDA-C cells (Figure 3D, E). Overall, we can conclude that Sirt3 affects the metabolic activity and the expression of all studied proteins involved in the metabolism of MDA-MB-231 cells, whereas hyperoxic treatment impacts the expression of Ldh-A and Sirt1 proteins.
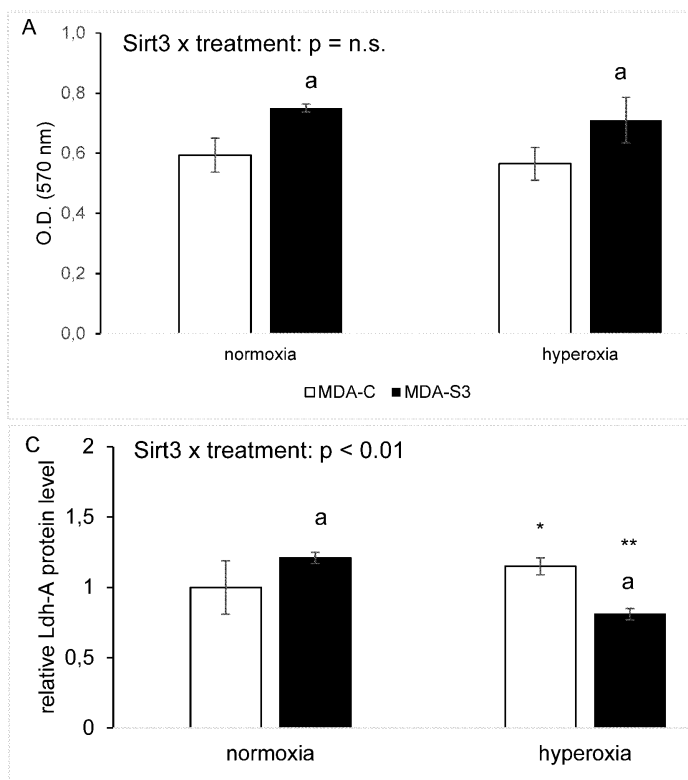

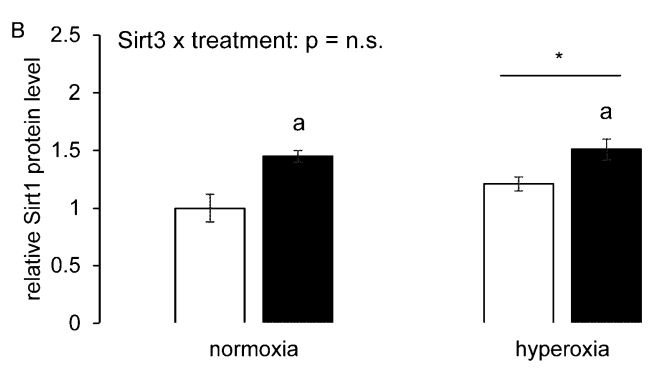

口MDA-C $\square$ MDA-S3

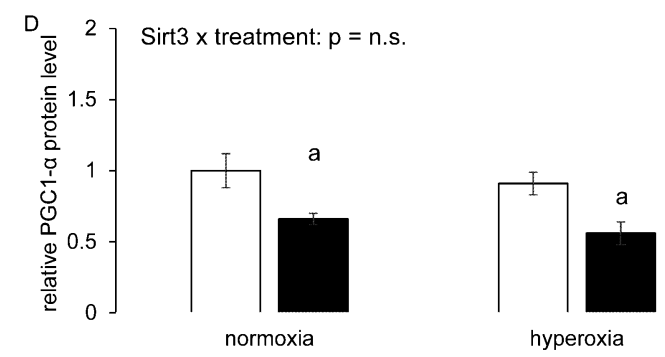

口MDA-C $\square$ MDA-S3

口MDA-C $\square$ MDA-S3

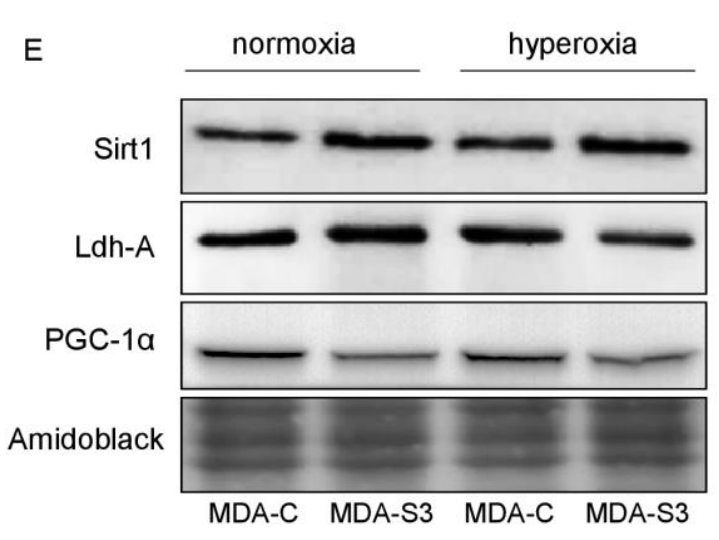

Figure 3. Overexpressed Sirt3 affects metabolic activity and proteins involved in metabolism with the reverse effect of hyperoxic treatment on Ldh-A expression in MDA-MB-231 cells. (A) Metabolic activity of MDA-C and MDA-S3 cells under normoxic and hyperoxic conditions measured by MTT test: MDA-S3 vs. MDA-C $\left({ }^{a} p<0.001\right)$. (B) 
Densitometry analysis of Sirt1 protein expression in MDA-C and MDA-S3 cells under normoxic and hyperoxic conditions: MDA-S3 vs. MDA-C $\left({ }^{a} p<0.01\right)$, hyperoxia vs. normoxia $\left({ }^{*} p<0.05\right)$. (C) Densitometry analysis of Ldh-A protein expression in MDA-C and MDA-S3 cells under normoxic and hyperoxic conditions: MDA-S3 vs. MDA-C $\left({ }^{a} p<0.01\right), M D A-C$ hyperoxia vs. normoxia ( $\left.{ }^{*} p<0.05\right), M D A-S 3$ hyperoxia vs. normoxia $\left({ }^{* *} p<0.01\right)$. (D) Densitometry analysis of PGC-1 $\alpha$ protein expression in MDA-C and MDA-S3 cells under normoxic and hyperoxic conditions: MDA-S3 vs. MDA-C ( $\left.{ }^{a} p<0.01\right)$. Data are presented as mean \pm S.D. Experiments were repeated at least three times and representative data are shown. (E) Immunoblots of Sirt1, Ldh-A and PGC-1 $\alpha$ protein expression in MDA-C and MDA-S3 cells under normoxic and hyperoxic conditions. Amido black was used as a loading control.

Overexpressed Sirt3 is not associated with a hyperoxic effect on ROS production and antioxidative enzymes in MDA-MB-231 cells. Since hyperoxia is a well-known inducer of ROS [24], mitochondrial (mtROS) and cytosolic ROS (cytoROS) production was analyzed using MitosoxRed and DHE dyes, respectively. We observed that MDA-S3 cells had higher mtROS in both normoxia and hyperoxia (Figure 4A), with no change in cytoROS compared to control cells (Figure 4B). Hyperoxic treatment increased the production of mtROS (Figure 4A) and cytoROS (Figure 4B) in both MDA-C and MDA-S3 cells. Due to the observed higher levels of ROS induced by either Sirt3 or hyperoxic treatment, we also analyzed the antioxidative status of transfected cells. MnSOD (SOD2) and CuZnSOD (SOD1) activities were analyzed, as well as the expression of SOD2 and catalase (Cat) proteins. SOD2 is one of the main targets of Sirt3 which is deacetylated and activated in response to oxidative stress [25]. Its activity (Figure 4C) and expression (Figure 4D, G) showed to follow the same pattern: increase in MDA-S3 cells in both normoxia and hyperoxia compared to MDA-C cells, and decrease upon hyperoxic treatment in both MDA-C and MDA-S3 cells. On the other hand, SOD1 activity was lower in MDA-S3 cells in both normoxia and hyperoxia, and was even lower in both MDA-C and MDAS3 cells upon hyperoxia (Figure 4E). In the case of Cat, only MDA-S3 cells had increased Cat expression in both normoxia and hyperoxia compared to MDA-C cells (Figure 4F, G). Overall, we can conclude that Sirt3 induces mtROS, as well as the expression and/or activity of several proteins involved in antioxidative response, whereas the hyperoxic treatment increases cellular ROS and decreases antioxidative response regardless of the Sirt3 expression. 


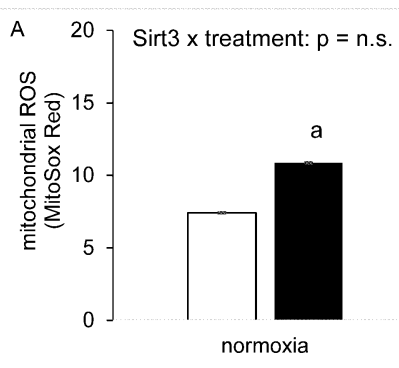

口MDA-C $\because$ MDA-S3

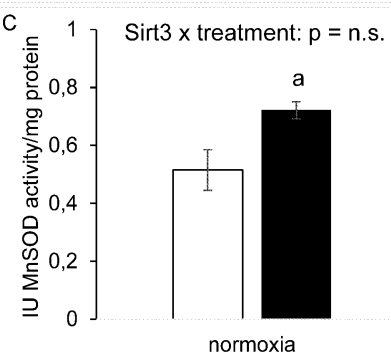

口MDA-C =MDA-S3

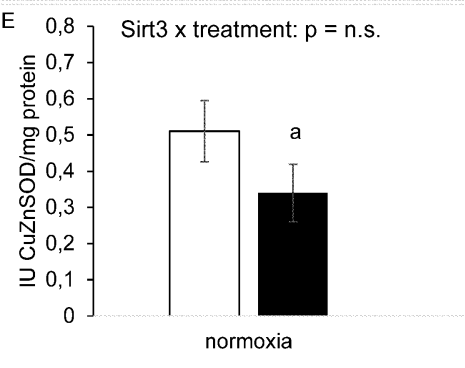

口MDA-C MDA-S3

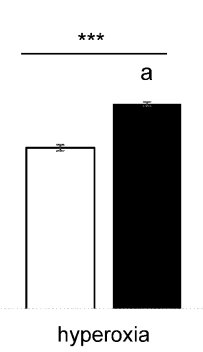

hyperoxia

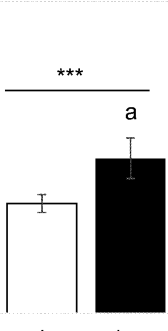

hyperoxia

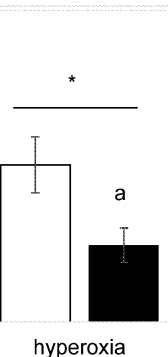

hyperoxia

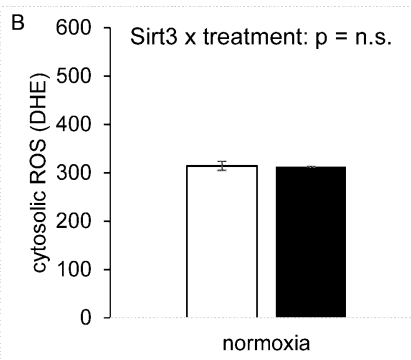

口MDA-C -MDA-S3

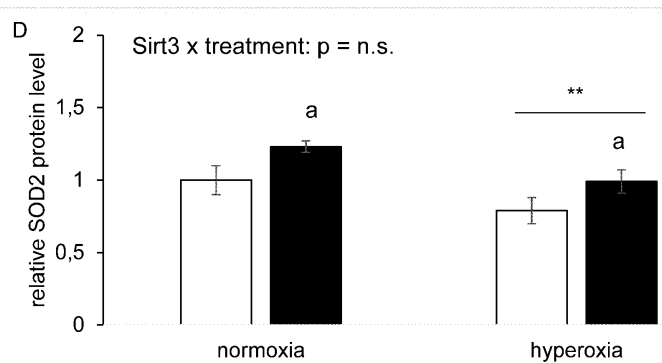

口MDA-C =MDA-S3

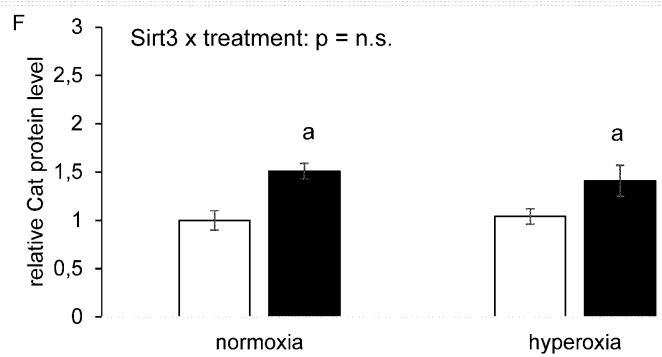

口MDA-C $=$ MDA-S3

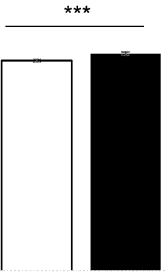

hyperoxia

hyperoxia

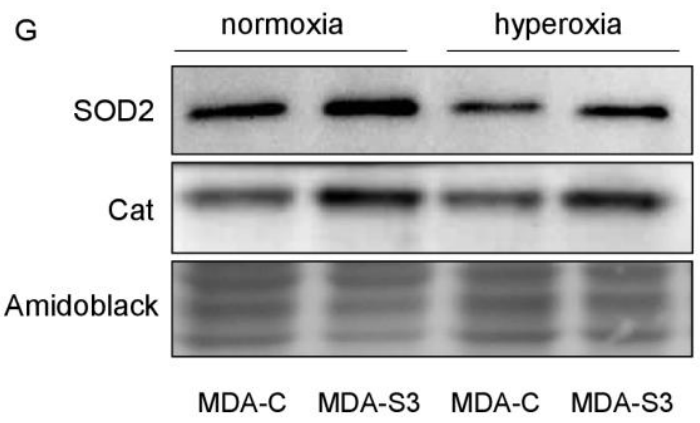

Figure 4. Overexpressed Sirt3 is not associated with a hyperoxic effect on ROS production and antioxidative enzymes in MDA-MB-231 cells. (A) Mitochondrial ROS production in MDA-C and MDA-S3 cells under normoxic and hyperoxic conditions measured with MitosoxRed dye: MDA-S3 vs. MDA-C ( $\left.{ }^{a} p<0.001\right)$, hyperoxia vs. normoxia (*** $p<0.001)$. (B) Cytosolic ROS production in MDA-C and MDA-S3 cells under normoxic and hyperoxic conditions measured with DHE dye: hyperoxia vs. normoxia ( $\left.{ }^{* *} p<0.001\right)$. (C) MnSOD (SOD2) activity in MDA-C and MDA-S3 cells under normoxic and hyperoxic conditions: MDA-S3 vs. MDA-C ( $\left.{ }^{\mathrm{a}} \mathrm{p}<0.001\right)$, hyperoxia vs. normoxia $(* * * p<0.001)$. (D) SOD2 protein expression in MDA-C and MDA-S3 cells under normoxic and hyperoxic conditions: MDA-S3 vs. MDA-C ( $\left.{ }^{a} p<0.05\right)$, hyperoxia vs. normoxia $\left({ }^{* * *} p<0.01\right)$. (E) CUZnSOD (SOD1) activity in MDA-C and MDA-S3 cells under 
normoxic and hyperoxic conditions: MDA-S3 vs. MDA-C ( $\left.{ }^{a} \mathrm{p}<0.001\right)$, hyperoxia vs. normoxia $\left({ }^{*} p<0.05\right)$. (F) Catalase (Cat) protein expression in MDA-C and MDA-S3 cells under normoxic and hyperoxic conditions: MDA-S3 vs. MDA-C ( $\left.{ }^{\mathrm{a} p}<0.01\right)$. Data are presented as mean \pm S.D. Experiments were repeated at least three times and representative data are shown. (G) Immunoblots of SOD2 and Cat protein expression in MDA-C and MDA-S3 cells under normoxic and hyperoxic conditions. Amido black was used as a loading control.

Both overexpressed Sirt3 and hyperoxic treatment increase the expression of $\mathrm{YH} 2 \mathrm{~A} . \mathrm{X}$ in MDA-MB-231 cells. Following the observed higher ROS upon either Sirt3 or hyperoxic treatment and induced antioxidative enzyme system in MDA-S3 cells in both normoxia and hyperoxia, analysis of $\mathrm{YH} 2 \mathrm{~A} . \mathrm{X}$ (a marker of double-strand DNA (dsDNA) breaks [26]) protein expression in MDA-C and MDA-S3 cells was performed. A significant increase of dsDNA breaks was observed upon Sirt3 expression in both normoxia and hyperoxia compared to MDA-C cells and was further induced upon hyperoxic treatment in both MDA-C and MDA-S3 cells (Figure 5A). Cellular localization and the strength of the $\mathrm{\gamma H} 2 \mathrm{~A}$.X fluorescence signal were also analyzed by immunofluorescence using a confocal microscope. Stronger intensity of the YH2A.X fluorescence signal was observed in both MDA-C and MDA-S3 cells after hyperoxic treatment, with an even stronger signal in the MDA-S3 cells (Figure 5B). Overlay image confirmed the localization of $\gamma \mathrm{H} 2 \mathrm{~A}$.X in the nucleus. Thus, we can conclude that both Sirt3 and hyperoxia increase the formation of dsDNA breaks.
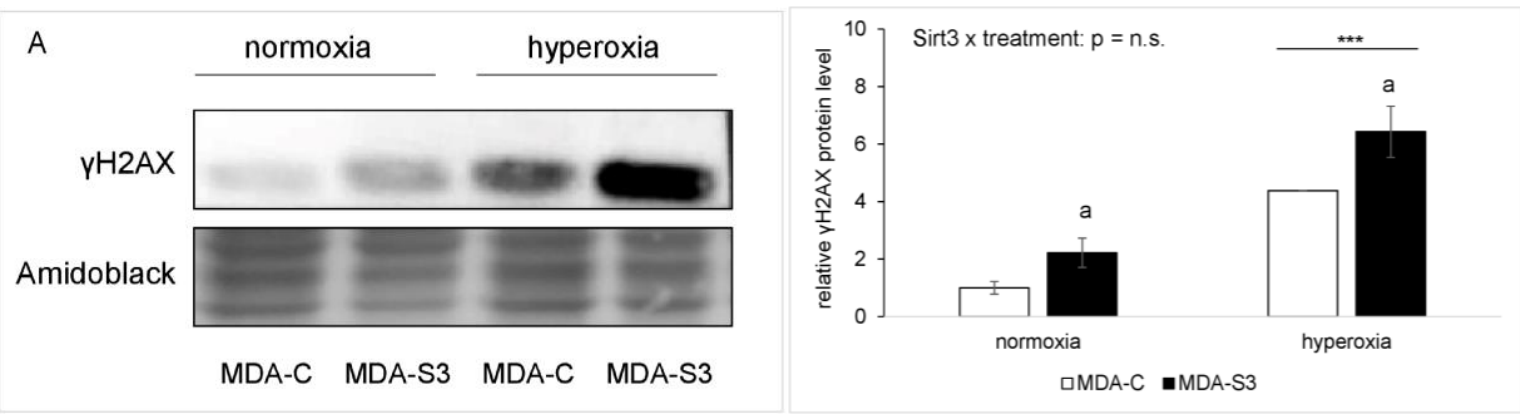


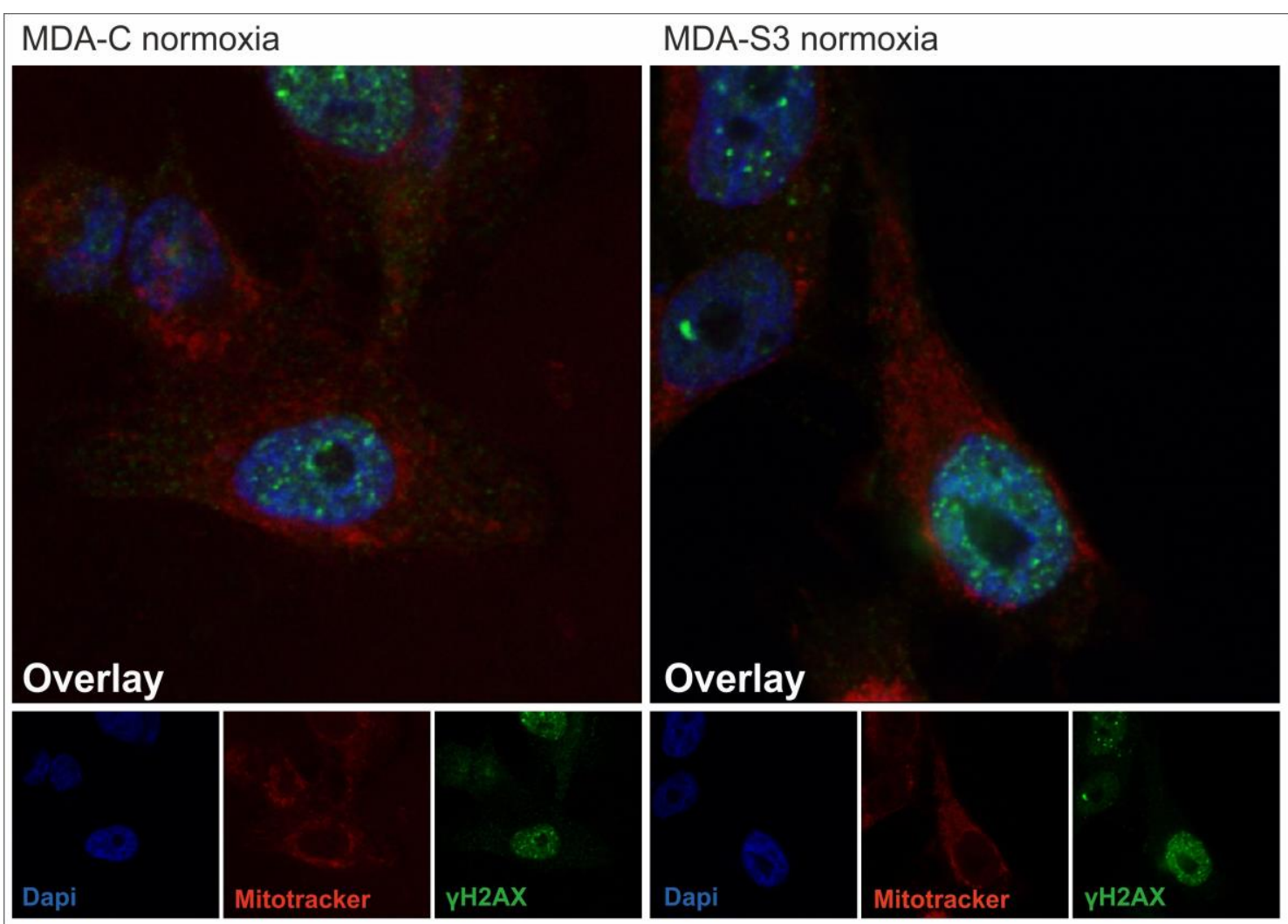

MDA-C hyperoxia

MDA-S3 hyperoxia

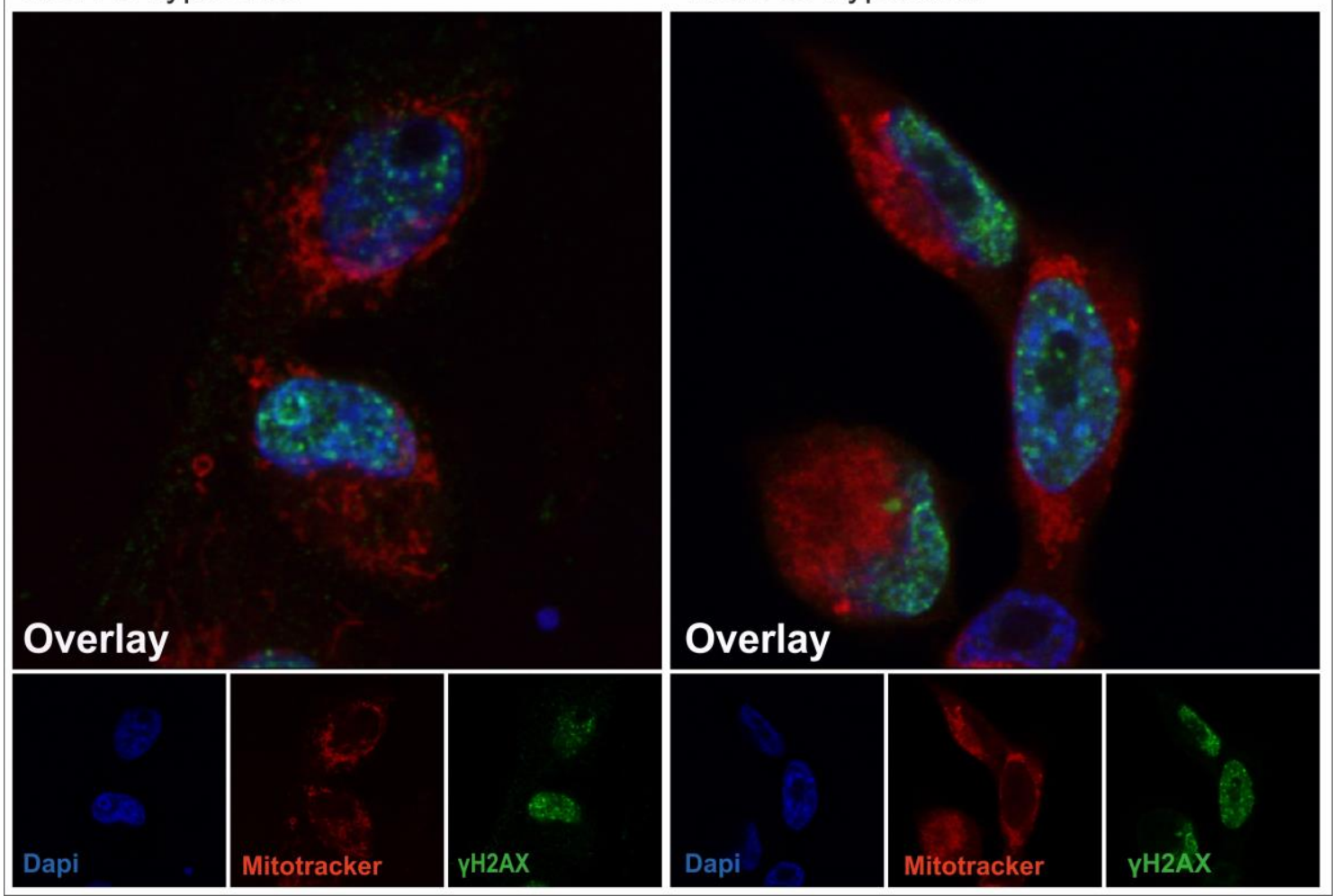

Figure 5. Both overexpressed Sirt3 and hyperoxic treatment increase the expression of $\mathrm{YH2A.X}$ in MDA-MB-231 cells. (A) Densitometry analysis and immunoblots of $\mathrm{YH} 2 \mathrm{~A}$.X protein expression in MDA-C and MDA-S3 cells under normoxic and hyperoxic 
conditions: MDA-S3 vs. MDA-C ( $\left.{ }^{a} p<0.05\right)$, hyperoxia vs. normoxia $\left({ }^{* * *} p<0.001\right)$. Data are presented as mean \pm S.D. Amido black was used as a loading control. Experiments were repeated at least three times and representative data are shown. (B) Representative confocal image of $\mathrm{\gamma H} 2 \mathrm{~A} . \mathrm{X}$ (green) in nucleus (blue; DAPI). Mitochondria were detected with MitoTracker Deep Red. Overlay image shows an overlap of the $\gamma \mathrm{H} 2 \mathrm{~A}$. $\mathrm{X}$ and nuclear staining.

344 Overexpressed Sirt3 increases the mitotic index in normoxia, whereas both Sirt3 and 345 hyperoxia increase timp-1 gene expression and reduce colony-forming capacity in MDA-MB-

346231 cells. Since we observed higher expression of $\mathrm{YH} 2 \mathrm{~A} . \mathrm{X}$ in MDA-S3 cells and upon the 347 hyperoxic treatment, and DNA damage is known to often cause cell cycle arrest and no proper 348 cell division [27], we next determined mitotic index, timp-1 gene expression and colony349 forming ability of the transfected cells. MDA-S3 cells showed a higher mitotic index in 350 normoxic conditions than control cells, whereas hyperoxia reversed this effect of Sirt3 and reduced mitotic index in both control and MDA-S3 cells (Figure 6A). Due to the observed combined negative effect of Sirt3 and hyperoxia on the mitotic index, we also determined the expression of the timp-1 gene, which is known as an inhibitor of metalloproteinases and its increased expression is associated with consequential reduction of cellular invasiveness in MDA-MB-231 cells-derived tumors $[28,29]$. The results of the GPCR analysis showed that MDA-S3 cells have a 2-fold higher expression of the timp1 gene in normoxia and 5-fold higher in hyperoxia compared to MDA-C (Figure 6B). Hyperoxia also induced its expression in MDA$C$ cells compared to normoxia (1,5-fold). The pattern observed in mitotic index analysis was also expected in a colony-forming assay, however, Sirt3 expression reduced colony-forming ability in both normoxic and hyperoxic conditions, and hyperoxia markedly amplified this negative effect in both MDA-C and MDA-S3 cells (Figure 6C, D). Overall, we can conclude that both Sirt3 and hyperoxia, alone or in combination, negatively impact the proliferation characteristics of MDA-MB-231 cells. 

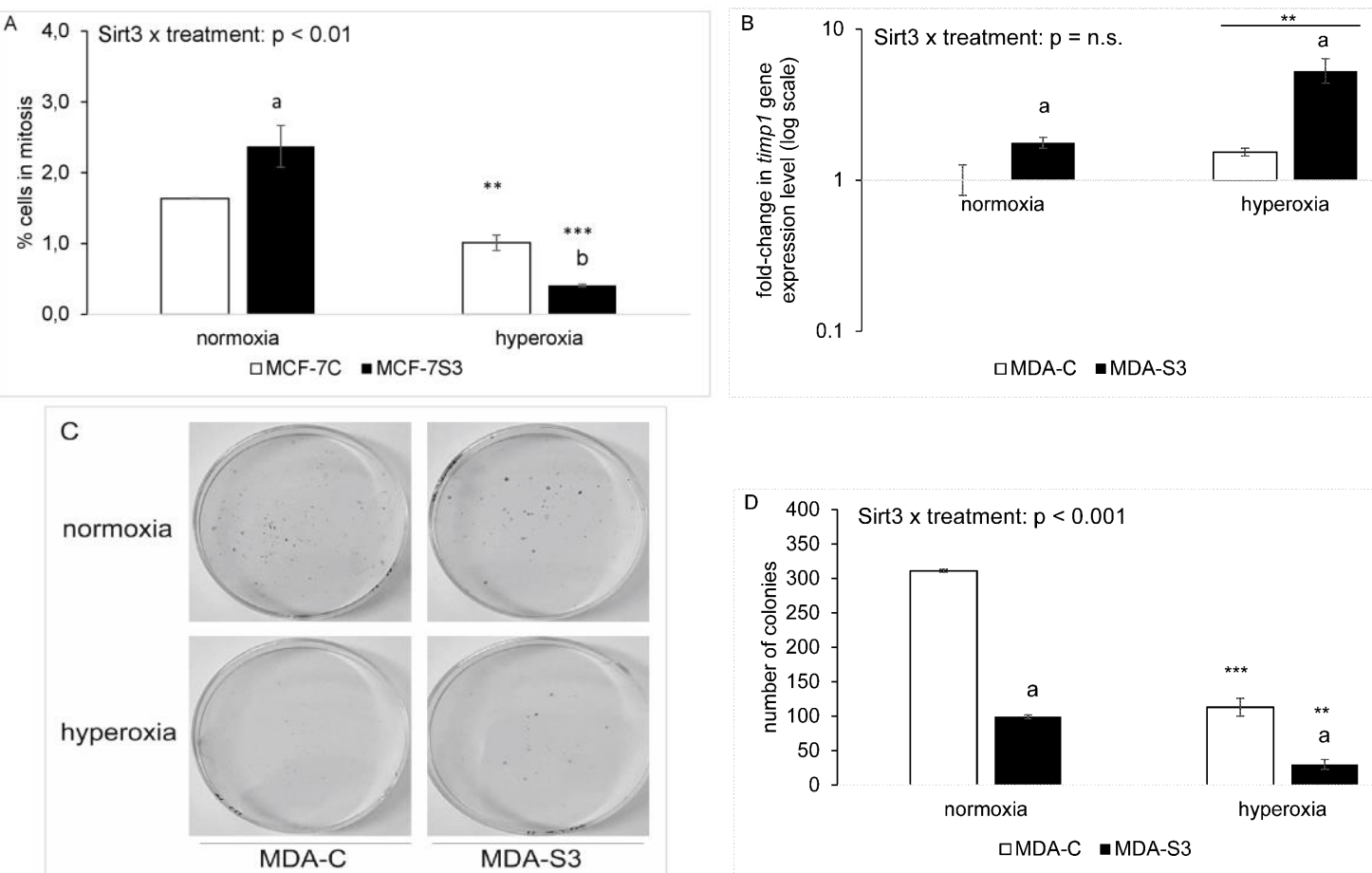

Figure 6. Overexpressed Sirt3 increases the mitotic index in normoxia, whereas both Sirt3 and hyperoxia reduce colony-forming capacity and increase timp-1 gene expression in MDA-MB-231 cells. (A) Percentage of MDA-C and MDA-S3 cells in mitosis (mitotic index) under normoxic and hyperoxic conditions: MDA-S3 vs. MDA-C $\left({ }^{a} p<0.01,{ }^{b} p<0.05\right), M D A-C$ hyperoxia vs. normoxia ( $\left.{ }^{* *} p<0.01\right)$, MDA-S3 hyperoxia vs. normoxia ( $\left.{ }^{* * *} p<0.001\right)$. (B) Real-time PCR analysis of timp-1 gene expression in MDA$C$ and MDA-S3 cells under normoxic and hyperoxic conditions: MDA-S3 vs. MDA-C $\left({ }^{a} p<0.01\right)$, hyperoxia vs. normoxia $\left({ }^{* *} p<0.01\right)$. Data are presented as fold-change \pm S.E., and normalized to control group. $\beta$-actin was used as the endogenous control. (C) Representative plates of colonies stained with crystal violet. (D) The number of colonies formed by MDA-C and MDA-S3 cells under normoxic and hyperoxic conditions: MDA-S3 vs. MDA-C $\left({ }^{\mathrm{a}} \mathrm{p}<0.001\right)$, MDA-C hyperoxia vs. normoxia $(* * * p<0.001)$, MDA-S3 hyperoxia vs. normoxia $(* * p<0.01)$. Data for $(A)$ and $(D)$ are presented as mean \pm S.D. Experiments were repeated at least three times and representative data are shown.

Overexpressed Sirt-3 is associated with hyperoxia-induced apoptosis in MDA-MB-231 cells.

Due to the inhibitory effect of Sirt3 and hyperoxia on mitotic index and CFU analysis, and increased DNA damage, we aimed to analyze apoptosis rate with an Annexin V-FITC Apoptosis Kit in transfected cells as a possible outcome of all the observed parameters. The FACS analysis showed an increased percentage of apoptotic cells in the MDA-S3 cells compared to MDA-C cells in normoxia (Figure 7A, B), which was further enhanced by hyperoxic treatment. Interestingly, MDA-C cells did not show an increase in the number of apoptotic cells after hyperoxic treatment. Thus, we can conclude that hyperoxia-induced apoptosis is associated with overexpressed Sirt-3 in MDA-MB-231 cells. 

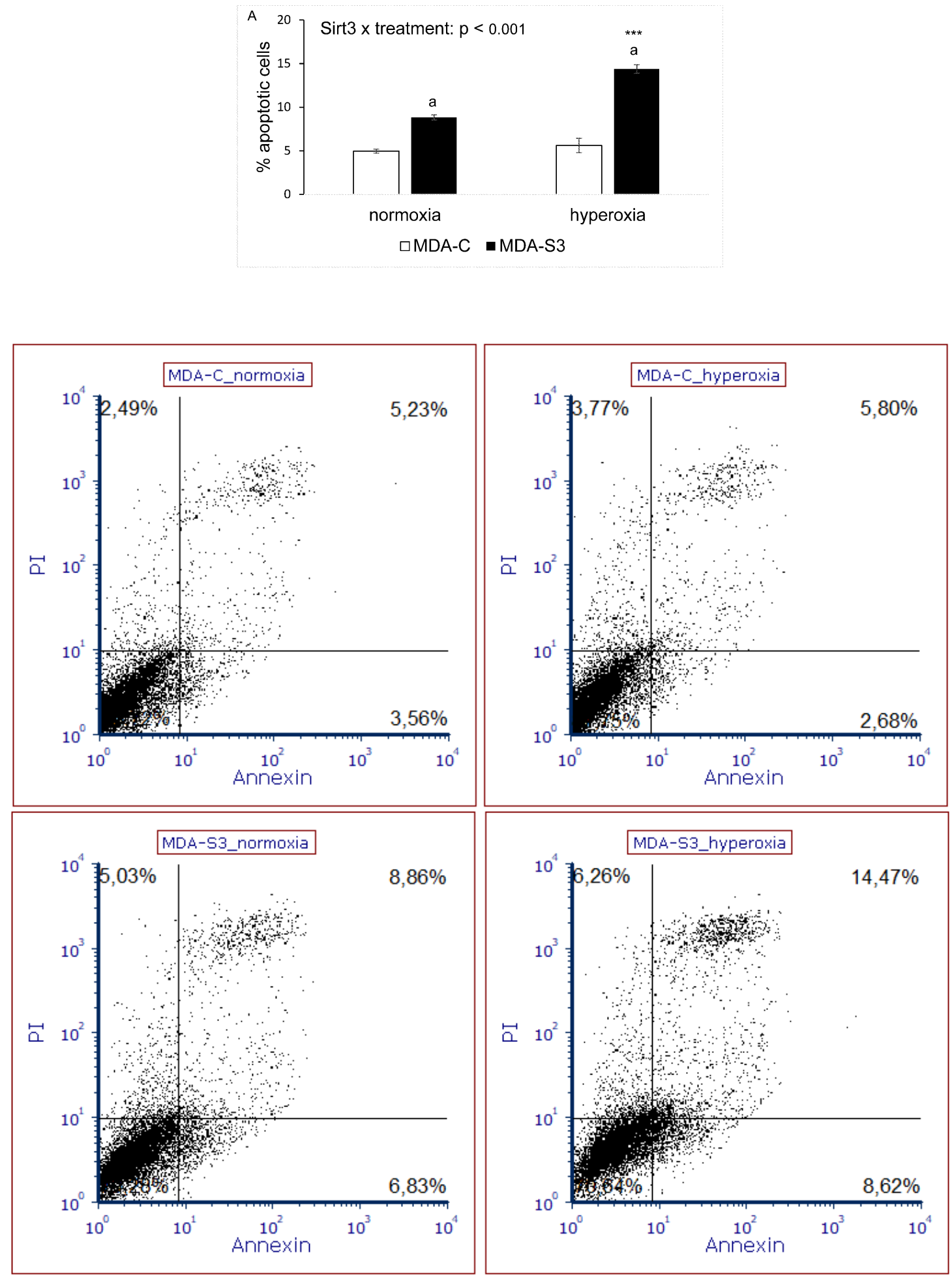

Figure 7. Overexpressed Sirt-3 is associated with hyperoxia-induced apoptosis in MDA-MB-231 cells. (A) Percentage of MDA-C and MDA-S3 cells in late apoptosis under normoxic and hyperoxic conditions: MDA-S3 vs. MDA-C ( $\left.{ }^{a} p<0.001\right)$, MDA-S3 hyperoxia vs. normoxia $(* * * p<0.001)$. Data are presented as mean \pm S.D. Experiments were repeated at least three times and representative data are shown. (B) Dot plot diagrams of MDA-C and MDA-S3 cells in apoptosis under normoxic and hyperoxic conditions quantified by FACS, after Annexin V-FITC and PI labelling: intact cells in the lower-left 

cells in the upper-right quadrant, and necrotic cells in the upper-left quadrant. Experiments were repeated at least three times and representative data are shown.

\section{Overexpressed Sirt3 and hyperoxia are associated with formation of multinucleated cells in}

403 MDA-MB-231 cells. Since MDA-S3 cells in normoxia had higher mitotic index but lower CFU, 404 this prompted us to look into cellular morphology in more detail. For this reason, nuclei of 405 live cells were stained with Hoechst and mitochondria with NAO. We noticed the presence of 406 multinucleated cells (MNCs), i.e. giant cells with three or more nuclei in the MDA-S3 cells 407 (Figure 8A). We also analyzed morphology of the colonies in both normoxic and hyperoxic 408 conditions formed in the CFU assay, and numerous MNCs were observed in MDA-S3 cells, 409 which were even more frequent upon hyperoxic conditions (Figure 8B). On the other hand, 410 we could barely observe MNCs in the MDA-C cells. Thus, we can conclude that Sirt3 and 411 hyperoxia are associated with formation of MNCs in MDA-MB-231 cell line.

412 A
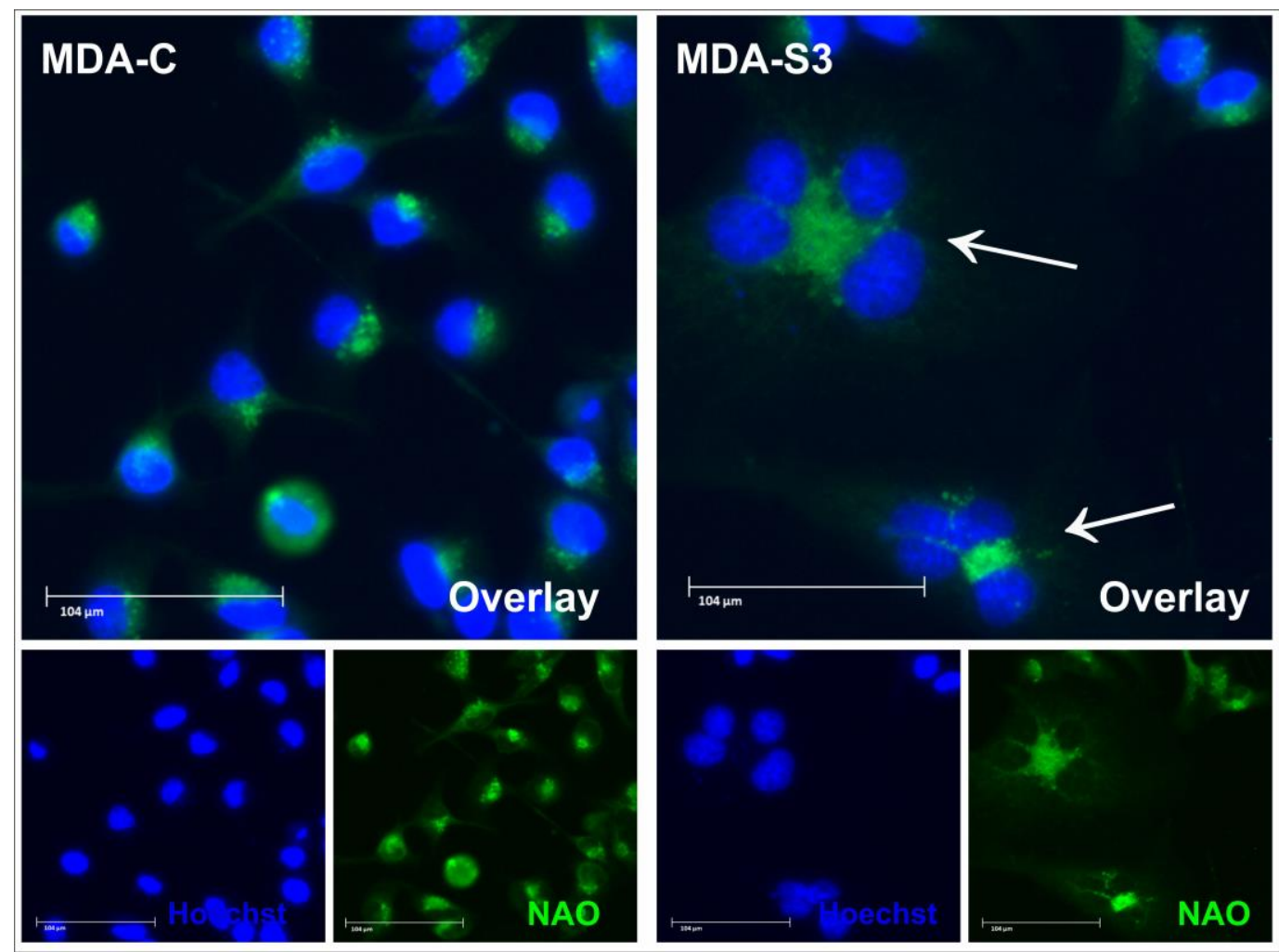


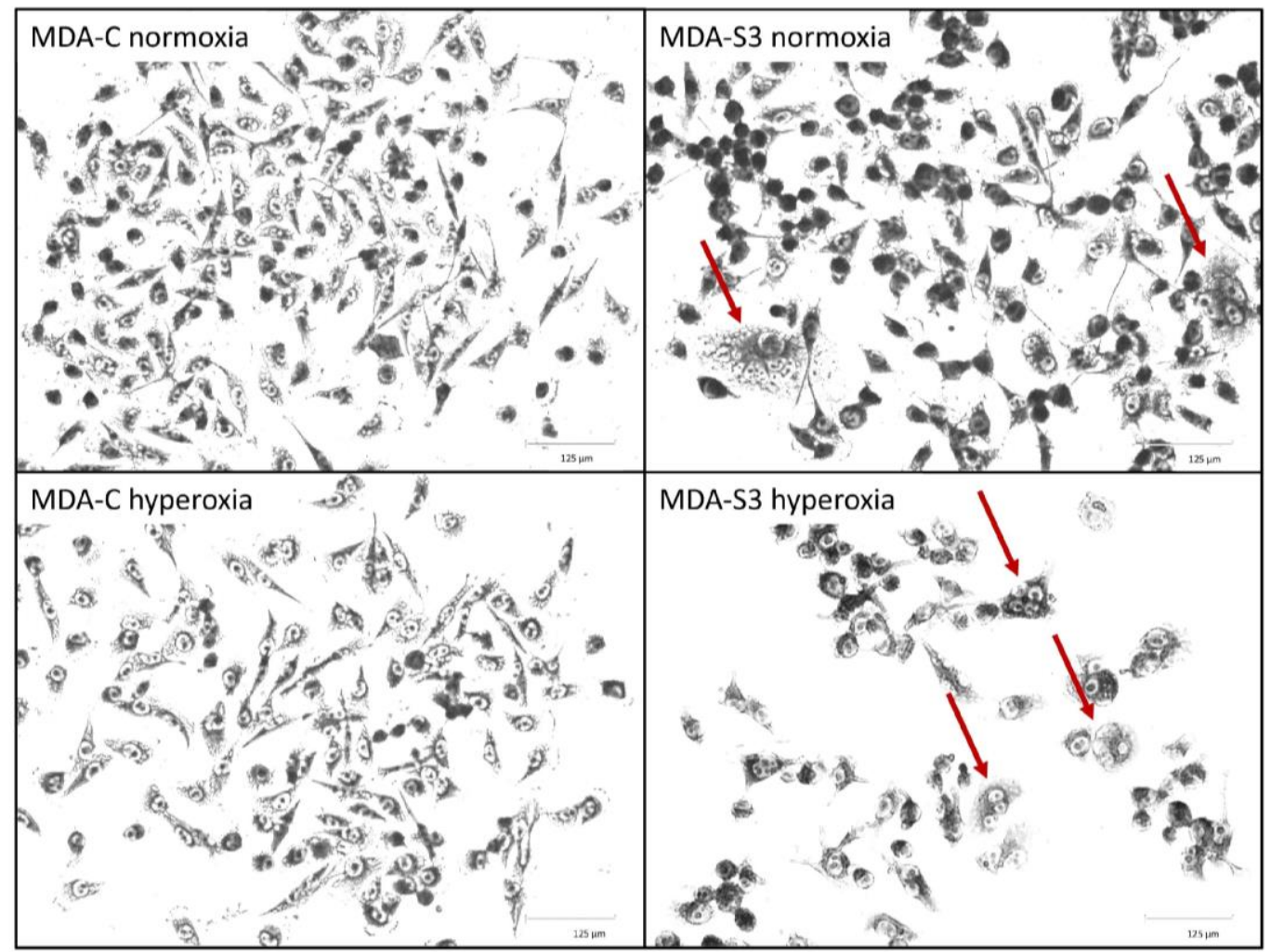

Figure 8. Overexpressed Sirt3 and hyperoxia are associated with formation of multinucleated cells in MDA-MB-231 cells. (A) Fluorescence microscopy images of live MDA-C and MDA-S3 cells in normoxic conditions. Nuclei were detected with Hoechst (blue), and mitochondria with NAO (green). Multinucleated cells in MDA-S3 are marked with white arrows. (B) Morphology of the MDA-C and MDA-S3 cells under normoxic and hyperoxic conditions in the colony forming assay. Multinucleated cells in MDA-S3 are marked with red arrows.

Overall, although Sirt3 improved several mitochondrial properties, such as mitochondrial mass and potential, metabolic activity, Ldh-A expression and antioxidative defense (SOD2, Cat), it also increased mtROS and timp-1 expression, thus diminishing the ability of the MDAMB-231 cells to form colonies, induced formation of multinucleated cells as well as apoptotic cell death. These effects were even more pronounced upon hyperoxic treatment, thus pointing towards combined negative effect of Sirt3 and hyperoxia on MDA-MB-231 cells.

\section{Discussion}

431 Most breast cancer cell lines have very low level of mitochondrial protein Sirt3, which is known to have either oncogenic or tumor-suppressive role in different types of cancer [8]. This prompted us to explore the role of Sirt3 in TNBC cell line MDA-MB-231 upon elevated oxidative stress, i.e. hyperoxia $\left(95 \% \mathrm{O}_{2}\right)$. To carry out this study, we transfected the cells with the plasmid carrying the sirt3 gene and analyzed its effect on various mitochondrial and tumorigenic characteristics in normoxia and hyperoxia. 
Sirt3 overexpressed in MDA-MB-231 cells was increased upon hyperoxic treatment (Figure 1), most likely due to the elevated oxidative stress which resulted in enhancement of Sirt3 activity, deacetylation of mitochondrial enzymes involved in defense against oxidative stress and consequent protection from ROS, as demonstrated earlier [30]. To defend the cells from oxidative stress, the liposomes containing both Cat and SOD2 showed to have greater protection compared to liposomes containing only one of these two enzymes [31]. Previous studies showed that an increase in ROS stimulates sirt3 transcription, leading to posttranslational regulation and activation of SOD2 [25]. This is confirmed in our study by the increased mtROS and elevated SOD2 level in MDA-S3 cells (Figure 4A, C, D). Although cancer cells usually have increased ROS compared to normal cells, elevating intracellular ROS to toxic levels can activate various ROS-induced cell death pathways, or inhibit cancer cell resistance to chemotherapy (reviewed in [32]). Also, in the apoptosis pathway, mitochondrial potential is decreased, i.e. mitochondrial membrane permeability is increased, and pro-apoptotic factors are released from the mitochondria (reviewed in [32]). Since we also observed lower mitochondrial potential upon hyperoxic treatment compared to normoxia in both MDA-C and MDA-S3 cells (Figure 2B) as well as higher apoptosis rate in MDA-S3 cells (Figure 7), we can assume that the hyperoxia-induced high cytosolic and mtROS levels (Figure 4A, B) are indeed toxic for these cells, resulting in disruption of the whole antioxidant system, as observed through lower SOD2 and SOD1 activity upon hyperoxia (Figure 4C, E). The lower activity of the antioxidant enzyme SOD1 in MDA-S3 cells (Figure 4E) is in correlation with a previous study which showed that loss of SOD2 correlates with the overexpression of SOD1 [33], as well as that SOD2 reduction in breast cancer occurs rapidly following oncogenic activation, which was in our study reverted by Sirt3 expression. The third antioxidative enzyme, Cat, has enhanced expression in MDA-S3 cells, independent of hyperoxic treatment (Figure 4F). There are indications that increased levels of Cat could contribute to the improved treatment of breast cancer [34]. Accordingly, the role of Sirt3 to enhance Cat expression may indicate its tumor suppressive role in these cells. Therefore, we can assume that higher expression of Cat and increased SOD2 activity, with lower activity of SOD1 contributes to the mitigation of tumorigenic characteristics of MDA-MB-231 cells upon Sirt3 expression, whereas hyperoxia induces toxic levels of ROS which disrupt antioxidative defense and mitochondrial potential and finally have pro-apoptotic effects.

468 The main source of ROS in cell is the mitochondrial respiratory chain which is also a "target" for the deleterious effects of ROS [35]. It is known that Sirt3 activates the respiratory complexes responsible for the formation of ROS [36], and that it regulates numerous mitochondrial enzymes involved in energy metabolism processes [37]. Thus, the increased metabolic activity which we observed in the MDA-S3 cells was expected (Figure 3A). Sirt3 is also involved in metabolism-related regulation of tumor cell growth, i.e. in most cases it reduces the so-called Warburg effect, a metabolic sign of many tumors defined as the aerobic glycolysis [38]. However, Warburg-promoting effects of this enzyme have also been confirmed (reviewed in [39]). Improved mitochondrial mass and potential (Figure 2), higher 
metabolic activity and Ldh-A expression as well as lower PGC-1 $\alpha$ expression (Figure $\mathbf{3}$ ) in the

478 MDA-S3 cells in normoxic conditions suggest that Sirt3 has a potential to induce the aerobic 479 glycolysis in these cells. Ldh-A is upregulated in many cancers and is important for tumor 480 initiation and progression [40]. On the other hand, lower Ldh-A expression (Figure 3C) 481 associated with higher mitochondrial mass but lower mitochondrial potential (Figure 2), 482 higher ROS production (Figure 4) and apoptosis rate upon combined hyperoxic treatment and 483 Sirt3 expression is in correlation with an earlier study showing that Ldh-A-silenced cells accelerate oxygen consumption and have higher ROS, swollen mitochondria, and finally undergo apoptosis [40]. Thus, the combined effect of hyperoxia and Sirt3 expression on the reduction of Ldh-A expression in MDA-MB-231 cells further indicates their tumor-suppressive effect. We also analyzed the expression of Sirt1, a protein involved in regulation of metabolic responses which can be found in both nucleus and cytoplasm [20]. Besides its role in metabolic regulation, Sirt1 was also shown to be directly related to the DNA damage response and can recruit repair proteins to dsDNA breaks sites and modulate their activity by deacetylating them [21]. Therefore, higher Sirt1 expression in MDA-S3 cells and upon hyperoxic treatment (Figure 3B, E), both of which are associated with higher ROS (Figure 4) and dsDNA damage (Figure 5), is in accordance to the mentioned role of Sirt1 in oxidative stress.

Although mitochondrial analyses indicate that Sirt3, as a mitochondrial protein, succeeds in improving mitochondrial function of the MDA-MB-231 cells, the cells ultimately fail to divide and create colonies (Figure 6C, D). This is most likely due to the excessive DNA damage (Figure 5) resulting from the high concentration of ROS (Figure 4A, B). Excessive DNA damage often causes cell cycle arrest and no proper exit from mitosis and cell division [27]. In hyperoxia, both MDA-C and MDA-S3 cells showed pronounced decline in proliferation potential, consistent with other results suggesting tumor suppressor effects of hyperoxia on breast cancer cells [41]. Hyperoxia studies to date have considered whether hyperoxia acts as a tumor promoter or suppressor. Because oxygen is required for all major wound healing processes, it was thought that hyperoxia would promote tumor growth, but recent studies confirmed the inhibitory effects of hyperoxia on tumors (reviewed in [41]). We also performed quantitative RT-PCR to examine the effect of Sirt3 on timp-1, a main inhibitor of matrix metalloproteinase 9 (MMP-9) which drives malignant progression and metastasis of basal-like TNBC [29,42]. We found that Sirt3, and especially under hyperoxia, increases timp1 gene expression (Figure 6B), which further confirms their tumor-suppressive role in MDAMB-231 cells, since more recent studies point towards inhibitory role of timp-1 on cellular invasiveness of these cells [28]. Since MDA-S3 cells showed reduced number of colonies, as well as further reduction of colony number upon hyperoxia, we monitored also the mitotic index. MDA-S3 cells showed higher mitotic index in normoxia (Figure 6A) whereas, in hyperoxia, it followed the CFU pattern. This may be explained by the very interesting observation in this study that Sirt3 expression induced formation of multinucleated cells (MNCs; Figure 8). A number of studies stress out the importance of understanding the cause 
517 of their appearance, especially because they can arise spontaneously in the TNBC with mutant

518 p53, and are metabolically very active (reviewed in [43]). Since in our study the MDA-S3 cells 519 had higher mitotic index but lower colony forming ability, the most probable scenario for the 520 formation of MNCs is the "mitotic slippage", a phenomenon in which cells, due to prolonged 521 mitotic arrest, exit mitosis without undergoing cytokinesis and become multinucleated 522 (reviewed in [44]). Stressful conditions, like hypoxia, ionizing radiation and chemotherapeutic 523 drugs increase the proportion of polyploidy and MNCs. p53 mutant cells often try to avoid 524 senescence and apoptosis by forming MNCs (reviewed in [44]). In our case, we did not 525 observe senescence phenotype in MDA-MB-231 cells (data not shown), but the apoptosis 526 analysis clearly showed that cells expressing Sirt3 have higher apoptosis rate (Figure 7), and 527 this effect is even more pronounced upon hyperoxic treatment. Therefore, we can assume 528 that some cells expressing Sirt3 enter apoptosis, whereas the other ones try to avoid 529 apoptosis by forming MNCs (Figure 8). As pointed earlier and showed also now here, these 530 MNCs appear to be physiologically stressed with elevated ROS and lower mitochondrial 531 membrane potential [45]. The fact that MDA-S3 cells exhibited such increase of MNCs points 532 toward negative effect of Sirt3 on these cells, which then consequently impacts their 533 proliferation.

\section{Conclusion}

535 Although Sirt3 appeared to improve mitochondrial properties by increasing mitochondrial 536 mass and potential, metabolic activity (Warburg effect) and antioxidative defense (SOD2, 537 Cat), it also increased mitochondrial ROS, induced DNA damage, timp-1 expression, formation 538 of multinucleated cells and apoptosis, and markedly reduced the proliferation of MDA-MB539231 cells. All these negative effects on the cells were even more evident upon hyperoxic 540 treatment, which also reversed some effects of Sirt3. Finally, it can be concluded that Sirt3 541 and hyperoxia have a common tumor suppressor effect in the MDA-MB-231 breast cancer 542 cell line, and that additional mechanisms that influence this role in tumor cell proliferation 543 and invasiveness reduction should be studied in TNBC.

\section{References}

545 [1] J. Ferlay, M. Colombet, I. Soerjomataram, T. Dyba, G. Randi, M. Bettio, A. Gavin, O.

[2] S. Hurvitz, M. Mead, Triple-negative breast cancer: Advancements in characterization and treatment approach, Curr. Opin. Obstet. Gynecol. 28 (2016) 59-69. https://doi.org/10.1097/GCO.0000000000000239.

552 [3] K.J. Chavez, S. V. Garimella, S. Lipkowitz, Triple negative breast cancer cell lines: One 
tool in the search for better treatment of triple negative breast cancer, Breast Dis. 32 (2010) 35-48. https://doi.org/10.3233/BD-2010-0307.

555 [4] D.J. Betteridge, What is oxidative stress?, in: Metabolism., 2000: pp. 3-8. https://doi.org/10.1016/S0026-0495(00)80077-3.

557 [5] P.D. Ray, B.W. Huang, Y. Tsuji, Reactive oxygen species (ROS) homeostasis and redox regulation in cellular signaling, Cell. Signal. 24 (2012) 981-990. https://doi.org/10.1016/j.cellsig.2012.01.008.

560

[6] E. Panieri, M.M. Santoro, ROS homeostasis and metabolism: A dangerous liason in

561 cancer cells, Cell Death Dis. 7 (2016) e2253. https://doi.org/10.1038/cddis.2016.105.

562 [7] D. Hanahan, R.A. Weinberg, Hallmarks of cancer: The next generation, Cell. 144 (2011) 646-674. https://doi.org/10.1016/j.cell.2011.02.013.

564 [8] M. Torrens-Mas, J. Oliver, P. Roca, J. Sastre-Serra, SIRT3: oncogene and tumor 565 suppressor in cancer, Cancers (Basel). $9 \quad$ (2017) 90. https://doi.org/10.3390/cancers9070090.

567 [9] A. Raa, C. Stansberg, V.M. Steen, R. Bjerkvig, R.K. Reed, L.E.B. Stuhr, Hyperoxia retards 568 growth and induces apoptosis and loss of glands and blood vessels in DMBA-induced rat mammary tumors, BMC Cancer. 7 (2007) 23. https://doi.org/10.1186/1471-24077-23.

571

[10] S.W. Kim, I.K. Kim, J.H. Ha, C.D. Yeo, H.H. Kang, J.W. Kim, S.H. Lee, Normobaric hyperoxia inhibits the progression of lung cancer by inducing apoptosis, Exp. Biol. Med. 24 (2018) 739-748. https://doi.org/10.1177/1535370218774737.

574

[11] K.Y. Sletta, M.K. Tveitarås, N. Lu, A.S.T. Engelsen, R.K. Reed, A. Garmann-Johnsen, L. Stuhr, Oxygen-dependent regulation of tumor growth and metastasis in human breast cancer xenografts, PLoS One. 12 (2017) e0183254. https://doi.org/10.1371/journal.pone.0183254. 
[13] H.J.M. Weir, J.D. Lane, N. Balthasar, SIRT3: a central regulator of mitochondrial adaptation in health and disease, genes and cancer. 4 (2013) 118-124. https://doi.org/10.1177/1947601913476949.

584

[14] J.M. Marcus, S.A. Andrabi, Sirt3 regulation under cellular stress: Making sense of the 585 ups and downs, Front. Neurosci. $12 \quad$ (2018) 799. https://doi.org/10.3389/fnins.2018.00799.

587

[15] V.A. Kostyuk, A.I. Potapovich, Mechanisms of the suppression of free radical overproduction by antioxidants, Front. Biosci. - Elit. 1 (2009) 179-188.

589

[16] M. Pinterić, I.I. Podgorski, S. Sobočanec, M. Popović Hadžija, M. Paradžik, A. Dekanić, M. Marinović, M. Halasz, R. Belužić, G. Davidović, A. Ambriović Ristov, T. Balog, De novo expression of transfected sirtuin 3 enhances susceptibility of human MCF-7 breast cancer cells to hyperoxia treatment, Free Radic. Res. 52 (2018) 672-684. https://doi.org/10.1080/10715762.2018.1462495.

[17] M. Pinterić, I.I. Podgorski, M.P. Hadžija, V. Filić, M. Paradžik, B.L.J. Proust, A. Dekanić, I. Ciganek, D. Pleše, D. Marčinko, T. Balog, S. Sobočanec, Sirt3 exerts its tumorsuppressive role by increasing p53 and attenuating response to estrogen in MCF-7 cells, Antioxidants. 9 (2020) 294. https://doi.org/10.3390/antiox9040294.

[18] W. Yang, K. Nagasawa, C. Münch, Y. Xu, K. Satterstrom, S. Jeong, S.D. Hayes, M.P. Jedrychowski, F.S. Vyas, E. Zaganjor, V. Guarani, A.E. Ringel, S.P. Gygi, J.W. Harper, M.C. Haigis, Mitochondrial sirtuin network reveals dynamic SIRT3-dependent deacetylation in response to membrane depolarization, Cell. 167 (2016) 985-1000. https://doi.org/10.1016/j.cell.2016.10.016.

603 [19] M.M. Hasan-Olive, K.H. Lauritzen, M. Ali, L.J. Rasmussen, J. Storm-Mathisen, L.H. 604 605 Bergersen, A Ketogenic diet improves mitochondrial biogenesis and bioenergetics via 606 the PGC1 $\alpha$-SIRT3-UCP2 axis, Neurochem. Res. 44 (2019) 22-37. https://doi.org/10.1007/s11064-018-2588-6.

607 [20] M. Tanno, J. Sakamoto, T. Miura, K. Shimamoto, Y. Horio, Nucleocytoplasmic shuttling 
of the NAD+-dependent histone deacetylase SIRT1, J. Biol. Chem. 282 (2007) 68236832. https://doi.org/10.1074/jbc.M609554200.

[21] D.K. Alves-Fernandes, M.G. Jasiulionis, The role of SIRT1 on DNA damage response and epigenetic alterations in cancer, Int. J. Mol. Sci. 20 (2019) 3153. https://doi.org/10.3390/ijms20133153.

613

[22] V. Ganapathy, M. Thangaraju, P.D. Prasad, Nutrient transporters in cancer: Relevance to Warburg hypothesis and beyond, Pharmacol. Ther. 121 (2009) 29-40. https://doi.org/10.1016/j.pharmthera.2008.09.005.

616

[23] H. Liang, W.F. Ward, PGC-1 $\alpha$ : A key regulator of energy metabolism, Adv. Physiol. Educ. 30 (2006) 145-151. https://doi.org/10.1152/advan.00052.2006.

618

[24] A. Gore, M. Muralidhar, M.G. Espey, K. Degenhardt, L.L. Mantell, Hyperoxia sensing: From molecular mechanisms to significance in disease, J. Immunotoxicol. 7 (2010) 239254. https://doi.org/10.3109/1547691X.2010.492254.

621

[25] Y. Chen, J. Zhang, Y. Lin, Q. Lei, K.L. Guan, S. Zhao, Y. Xiong, Tumour suppressor SIRT3 deacetylates and activates manganese superoxide dismutase to scavenge ROS, EMBO Rep. 12 (2011) 534-541. https://doi.org/10.1038/embor.2011.65.

624 [26] L.J. Kuo, L.X. Yang, $\mathrm{Y}$-H2AX- A novel biomaker for DNA double-strand breaks, In Vivo (Brooklyn). 22 (2008) 305-309.

[27] A. Mikhailov, R.W. Cole, C.L. Rieder, DNA damage during mitosis in human cells delays the metaphase/anaphase transition via the spindle-assembly checkpoint, Curr. Biol. 12 (2002) 1797-1806. https://doi.org/10.1016/S0960-9822(02)01226-5.

[28] J. Batra, J. Robinson, C. Mehner, A. Hockla, E. Miller, D.C. Radisky, E.S. Radisky, PEGylation extends circulation half-life while preserving in vitro and in vivo activity of tissue inhibitor of metalloproteinases-1 (TIMP-1), PLoS One. 7 (2012) e50028. https://doi.org/10.1371/journal.pone.0050028.

[29] R. Zhou, L. Xu, M. Ye, M. Liao, H. Du, H. Chen, Formononetin inhibits migration and invasion of MDA-MB-231 and 4T1 breast cancer cells by suppressing MMP-2 and MMP- 
9 through PI3K/AKT signaling pathways, Horm. Metab. Res. 46 (2014) 753-760. https://doi.org/10.1055/s-0034-1376977.

637

638

639

640

641

642

643

644

645

646

647

648

649

650

651

652

653

654

655

656

657

658

659

660

661

[30] A.S. Bause, M.C. Haigis, SIRT3 regulation of mitochondrial oxidative stress, Exp. Gerontol. 48 (2013) 634-639. https://doi.org/10.1016/j.exger.2012.08.007.

[31] B. Halliwell, J.M.C. Gutteridge, Free radicals in biology and medicine, 2015. https://doi.org/10.1093/acprof:oso/9780198717478.001.0001.

[32] S. Galadari, A. Rahman, S. Pallichankandy, F. Thayyullathil, Reactive oxygen species and cancer paradox: To promote or to suppress?, Free Radic. Biol. Med. 104 (2017) 144164. https://doi.org/10.1016/j.freeradbiomed.2017.01.004.

[33] L. Papa, M. Hahn, E.L. Marsh, B.S. Evans, D. Germain, SOD2 to SOD1 switch in breast cancer, J. Biol. Chem. 289 (2014) 5412-5416. https://doi.org/10.1074/jbc.C113.526475.

[34] F. Tas, H. Hansel, A. Belce, S. Ilvan, A. Argon, H. Camlica, E. Topuz, Oxidative stress in breast cancer, Med. Oncol. 22 (2005) 11-15. https://doi.org/10.1385/mo:22:1:011.

[35] M. Ott, V. Gogvadze, S. Orrenius, B. Zhivotovsky, Mitochondria, oxidative stress and cell death, Apoptosis. 12 (2007) 913-922. https://doi.org/10.1007/s10495-007-07562.

[36] B.H. Ahn, H.S. Kim, S. Song, H.L. In, J. Liu, A. Vassilopoulos, C.X. Deng, T. Finkel, A role for the mitochondrial deacetylase Sirt3 in regulating energy homeostasis, Proc. Natl. Acad. Sci. U. S. A. $105 \quad$ (2008) 14447-14452. https://doi.org/10.1073/pnas.0803790105.

[37] J. Brenmoehl, A. Hoeflich, Dual control of mitochondrial biogenesis by sirtuin 1 and $\begin{array}{lllll}\text { sirtuin } 3, \quad \text { Mitochondrion. } & 13 & \text { (2013) 755-761. }\end{array}$ https://doi.org/10.1016/j.mito.2013.04.002.

[38] L.W. Finley, A. Carracedo, J. Lee, A. Souza, A. Egia, J. Zhang, J. Teruya-Feldstein, P.I. Moreira, S.M. Cardoso, C.B. Clish, P.P. Pandolfi, M.C. Haigis, SIRT3 opposes reprogramming of cancer cell metabolism through HIF1 $\alpha$ destabilization, Cancer Cell. 

19 (2011) 416-428. https://doi.org/10.1016/j.ccr.2011.02.014.

663

664

665

666

667

668

669

670

671

672

673

674

675

676

677

678

679

680

681

682

683

684

685

686

687

[39] Z. Gaál, L. Csernoch, Impact of sirtuin enzymes on the altered metabolic phenotype of malignantly transformed cells, Front. Oncol. 10 (2020) 45. https://doi.org/10.3389/fonc.2020.00045.

[40] Z.Y. Wang, T.Y. Loo, J.G. Shen, N. Wang, D.M. Wang, D.P. Yang, S.L. Mo, X.Y. Guan, J.P. Chen, LDH-A silencing suppresses breast cancer tumorigenicity through induction of oxidative stress mediated mitochondrial pathway apoptosis, Breast Cancer Res. Treat. 131 (2012) 791-800. https://doi.org/10.1007/s10549-011-1466-6.

[41] Y. Chen, L.L. Fu, X. Wen, X.Y. Wang, J. Liu, Y. Cheng, J. Huang, Sirtuin-3 (SIRT3), a therapeutic target with oncogenic and tumor-suppressive function in cancer, Cell Death Dis. 5 (2014) e1047. https://doi.org/10.1038/cddis.2014.14.

[42] C. Mehner, A. Hockla, E. Miller, S. Ran, D.C. Radisky, E.S. Radisky, Tumor cell-produced matrix metalloproteinase 9 (MMP-9) drives malignant progression and metastasis of basal-like triple negative breast cancer, Oncotarget. 5 (2014) 2736-2749. https://doi.org/10.18632/oncotarget.1932.

[43] R. Mirzayans, B. Andrais, D. Murray, Roles of polyploid/multinucleated giant cancer cells in metastasis and disease relapse following anticancer treatment, Cancers (Basel). 10 (2018) E118. https://doi.org/10.3390/cancers10040118.

[44] D. Sinha, P.H.G. Duijf, K.K. Khanna, Mitotic slippage: an old tale with a new twist, Cell Cycle. 18 (2019) 7-15. https://doi.org/10.1080/15384101.2018.1559557.

[45] A. Parekh, S. Das, S. Parida, C.K. Das, D. Dutta, S.K. Mallick, P.H. Wu, B.N.P. Kumar, R. Bharti, G. Dey, K. Banerjee, S. Rajput, D. Bharadwaj, I. Pal, K. kumar Dey, Y. Rajesh, B.C. Jena, A. Biswas, P. Banik, A.K. Pradhan, S.K. Das, A.K. Das, S. Dhara, P.B. Fisher, D. Wirtz, G.B. Mills, M. Mandal, Multi-nucleated cells use ROS to induce breast cancer chemoresistance in vitro and in vivo, Oncogene. 37 (2018) 4546-4561. https://doi.org/10.1038/s41388-018-0272-6. 